\title{
Low-excitation atomic gas around evolved stars
}

\section{ISO observations of C-rich nebulae}

\author{
D. Fong ${ }^{1}$, M. Meixner ${ }^{1}$, A. Castro-Carrizo ${ }^{2}$, V. Bujarrabal ${ }^{2}$, W. B. Latter ${ }^{3}$, A. G. G. M. Tielens ${ }^{4}$, \\ D. M. Kelly ${ }^{5}$, and E. C. Sutton ${ }^{1}$ \\ 1 University of Illinois, 1002 W. Green St., Urbana, IL 61801, USA \\ 2 Observatorio Astonómico Nacional, Apartado 1143, 28800 Alcalá de Henares, Spain \\ 3 CalTech, SIRTF Science Center, MS 314-6, Pasadena, CA 91125, USA \\ 4 Kapteyn Astronomical Institute, PO Box 800, 9700 AV Groningen, The Netherlands \\ 5 University of Arizona, Steward Observatory, Tucson, AZ 85721, USA
}

Received 11 July 2000 / Accepted 24 November 2000

\begin{abstract}
We present ISO LWS and SWS spectra of far-infrared (FIR) atomic fine structure lines in 12 carbonrich evolved stars including asymptotic giant branch (AGB) stars, proto-planetary nebulae (PPNe) and planetary nebulae (PNe). The spectra include grating and Fabry-Perot measurements of the line emission of [O I], [C II], [SiI], [SiII], [SI], [FeI], [FeII], [NeII] and [NII]. Only 5 out of our 12 object sample have been detected in at least one of these FIR lines. When we include the 12 oxygen-rich evolved stars from Castro-Carrizo et al. (2001, Paper II), we find that atomic line emission is observed only in those sources in which the central star's $T_{\text {eff }} \geq$ $10000 \mathrm{~K}$. Above this cutoff, the number of detectable lines and the intensity of the line emission increase as $T_{\text {eff }}$ increases. These trends suggest that the atomic lines originate from photodissociation regions (PDRs). In general, the kinematics of the atomic gas, derived from line fits to the Fabry-Perot data, are comparable to the molecular expansion velocities. These kinematics are expected for atomic cooling lines associated with circumstellar PDRs. AFGL 618, however, appears exceptional with dual velocity components: a narrow component $\left(<20 \mathrm{~km} \mathrm{~s}^{-1}\right)$ that may be associated with a PDR, and a broad component $\left(\sim 66 \mathrm{~km} \mathrm{~s}^{-1}\right)$ that may be produced in post-shocked, accelerated gas. A new PDR code which properly treats enhanced carbon abundances was used to model the observations of our carbon-rich objects. The predicted line intensities agree reasonably well with the observations. Shock models, however, do not compare well with the observed line intensities. PDR mass estimates ranging from $\sim 0.01-0.2 M_{\odot}$ were derived from the $[\mathrm{C}$ II $] 158 \mu \mathrm{m}$ line emission. The atomic gas constitutes only a small fraction of the total mass for young planetary nebulae, but its importance grows significantly as the nebulae evolve. Our overall analysis shows that photodissociation, and not shocks, dominates the evolution of the circumstellar envelope by transforming the initially molecular asymptotic giant branch envelopes into the atomic gas found in proto-planetary and planetary nebulae.
\end{abstract}

Key words. atomic data - stars: AGB and post-AGB - stars: circumstellar matter - stars: mass-loss ISM: planetary nebulae

\section{Introduction}

During their last stages of evolution, low mass stars $\left(<8 M_{\odot}\right)$ lose most of their mass in the form of a cool, low velocity $\left(10 \mathrm{~km} \mathrm{~s}^{-1}\right)$ molecular wind on the Asymptotic Giant Branch (AGB). After this intensive mass loss drives the star off the AGB, the star moves to the left in the HR diagram, passing through the short-lived, proto-planetary

\footnotetext{
Send offprint requests to: D. Fong,

e-mail: d-fong@astro.uiuc.edu

* Based on observations with ISO, an ESA project with instruments funded by ESA Member States (especially the PI countries: France, Germany, the Netherlands and the United Kingdom) and with the participation of ISAS and NASA.
}

nebula (PPN) phase. As the central star becomes hotter, the ejected circumstellar envelope will first be photodissociated, and then photoionized to become a planetary nebula (PN) (e.g., Iben 1995). At some point during its evolution, the central star will start losing mass in a fast $\left(100-1000 \mathrm{~km} \mathrm{~s}^{-1}\right)$, but low density wind. This fast wind will drive fast shocks into the circumstellar shell (e.g., Kwok 2000). In the formation of a PN, both photodissociation and shocks can chemically transform molecular material into atomic gas, but the relative contribution of these two processes remains ill defined.

Many studies of the molecular and ionized gas in evolved stars have been published. In contrast, little work has been done on the low-excitation atomic gas in evolved 
stars. Nevertheless, atomic gas has been previously observed in evolved stars using a variety of techniques. Most relevant to our work is the detection of the $[\mathrm{C} \mathrm{I}]$ $610 \mu \mathrm{m}$ line, which arises from low-excitation atomic gas in a number of carbon-rich (C-rich) evolved stars. Knapp et al. (2000) suggest that the origin of the [C I] line emission in these evolved stars differs depending on its evolutionary stage. In the C-rich AGB star, IRC +10216, the [CI] arises from the photodissociation of the outer envelope by the interstellar radiation field (ISRF) (Keene et al. 1993). In the C-rich PPN, AFGL 2688, the [C I] emission emerges from shock dissociation of C-rich molecules (Young 1997). Once the star is hot enough, e.g. in the Crich PPNe AFGL 618 (Young 1997) and HD 44179 (Knapp et al. 2000), the [C I] line arises from photodissociation of the inner molecular envelope by the central star. In the case of evolved C-rich PNe, e.g. NGC 6720 (Bachiller et al. 1994), the photodissociation of CO is clearly the origin of the $\left[\mathrm{CI}_{\mathrm{I}}\right]$ line. The detection of the $\mathrm{H}$ I $21 \mathrm{~cm}$ line in several $\mathrm{PNe}$ have also been interpreted as photodissociation of the molecular envelope by the central star (Taylor et al. 1990). Finally, optical (e.g. Gustafsson et al. 1997) and UV (e.g. Johnson et al. 1995) spectroscopy of AGB stars suggest the presence of gas phase metal atoms ( $\mathrm{Na}, \mathrm{K}, \mathrm{Fe}, \mathrm{Mg}$ and $\mathrm{Si}$ ) in the circumstellar outflows of these stars. The spectra of these atoms have enabled studies of the stellar outflows. In PNe, optical spectroscopy of $\mathrm{NaI}$ is a sensitive tracer of neutral material even when molecular gas tracers, such as CO, are not detected (e.g. Dinerstein et al. 1995a).

These previous studies, however, do not directly measure the physical conditions of the low-excitation atomic gas and, thus, they cannot determine the relative importance of shocks versus photodissociation. Theoretical studies of photodissociation regions (PDRs; Tielens \& Hollenbach 1985a; hereafter TH85; Spaans et al. 1994; Latter \& Tielens in preparation) and J-shocked gas regions (Hollenbach \& McKee 1989; hereafter HM89) show that they are cooled primarily by the far-infrared (FIR) atomic fine structure lines of [O I], [C II], [C I], [Si I], [Si II], $[\mathrm{S} \mathrm{I}]$, [Fe I] and [Fe II]. C-shocked gas regions, although predominantly molecular, are also cooled by [OI] and [S I] lines (Kaufman \& Neufeld 1996). The relative intensities of these FIR cooling lines are different for shocks and PDRs. Another feature to distinguish between shocks and photodissociation is the kinematics of the line emission. Shock excited lines are significantly broadened by the shock accelerated gas. Hence, by observing these FIR lines we can investigate the physical conditions and the underlying process that heats these low-excitation atomic gas regions.

In order to measure these main cooling lines, observations must be conducted from the air or space. Using the Kuiper Airborne Observatory, Dinerstein et al. (1995b) detected these FIR lines in several PNe. In their survey of about $20 \mathrm{PNe}$ and PPNe in the [O I] $63 \mu \mathrm{m}$ line, nearly all moderately-bright $\mathrm{PNe}$ showed strong emission at [O I] $63 \mu \mathrm{m}$. However, the [O I] $63 \mu \mathrm{m}$ line was not detected in PPNe nor in extremely dense, young PNe (Dinerstein 1995a). The [O I] $146 \mu \mathrm{m}$ and [C II] $158 \mu \mathrm{m}$ lines were also measured in several $\mathrm{PNe}$ and used with the [OI] $63 \mu \mathrm{m}$ line to estimate the gas temperature and density. The strong emission lines arising from these $\mathrm{PNe}$ suggested the presence of dense $\left(>10^{3}-10^{4} \mathrm{~cm}^{-3}\right)$, warm (>500-1000 K) photodissociation regions.

The Infrared Space Observatory (ISO) satellite mission has permitted larger coherent studies of the FIR lines in evolved stars. The ISO Long Wavelength Spectrometer (LWS) and Short Wavelength Spectrometer (SWS) grating spectra have been used to survey a large number of evolved stars (e.g. Barlow 1999; Liu et al. 2001). Using these grating spectra, the detection of the [OI] 63 and $146 \mu \mathrm{m}$ and [C II] $158 \mu \mathrm{m}$ lines have been noted in NGC 7027 (Liu et al. 1996), and the [O I] $63 \mu \mathrm{m}$ and [C II] $158 \mu \mathrm{m}$ lines have been claimed to be detected in AFGL 618 (Herpin \& Cernicharo 2000) and found to be undetected in AFGL 2688 (Cox et al. 1996). However, little analysis of these lines has been done. The primary focus of some recent analysis on the line emission found in ISO LWS and SWS grating spectra has been on the molecular gas (e.g. Yan et al. 1999; Liu et al. 1996; Cernicharo et al. 1997) or on the highly ionized gas of the H II region (e.g. Feuchtgruber et al. 1997; Pequignot et al. 1997).

In this paper we present a systematic study of the lowexcitation atomic gas in evolved stars using ISO SWS and LWS spectra. Our study is distinct from previous studies in that the spectral resolution is sufficient to obtain the important kinematic information. Our sample of 24 objects spans the AGB, PPN and PN phases of evolution and includes an equal number of C-rich and oxygen-rich (O-rich) objects. AGB stars were included to investigate the contribution to the far-infrared lines from the PDR created in the outer envelope by the interstellar radiation field. While some of the PNe are known to have these lines, the PPNe are basically unstudied before our work. By using all three evolutionary stages, we can trace the transformation of the molecular gas into atomic gas.

Our work is divided into two papers: here in Paper I, we present the results for the 12 C-rich evolved stars, and in Paper II (this volume), we present the results for the 12 O-rich evolved stars. The separation of the sources was motivated by the different chemistry found in C-rich and O-rich PDRs which requires completely different models. In particular, for the C-rich evolved stars, a new PDR code that treats enhanced carbon abundances will be introduced.

\section{Observations and data analysis}

We observed FIR atomic fine structure lines in the 12 C-rich evolved stars: IRC +10216, IRAS 151945115, LP And, IRAS 22272+5435, AC Her, SAO 163075, AFGL 2688 (the Egg Nebula), AFGL 915 (the Red Rectangle), IRAS 21282+5050, AFGL 618, NGC 6720 (the Ring Nebula) and NGC 7027. The FIR lines include: [Ne II] $12.8 \mu \mathrm{m},[\mathrm{Fe}$ I] 24 and $35 \mu \mathrm{m}$, [S I] $25 \mu \mathrm{m},[\mathrm{Fe} I \mathrm{I}] 26$ and 
Table 1. Instrumental properties

\begin{tabular}{|c|c|c|c|c|c|c|}
\hline $\mathrm{AOT}$ & \multicolumn{2}{|c|}{$\begin{array}{l}\text { Line } \\
(\mu \mathrm{m})\end{array}$} & $\begin{array}{l}\text { Resolution } \\
\text { Element } \\
(\mu \mathrm{m})\end{array}$ & $\begin{array}{c}\text { Resolving } \\
\text { Power } \\
\text { (approx) }\end{array}$ & $\begin{array}{c}\text { Spectral } \\
\text { Resolution } \\
\left(\mathrm{km} \mathrm{s}^{-1}\right)\end{array}$ & $\begin{array}{c}\text { Beam } \\
\text { Size } \\
\left({ }^{\prime \prime}\right)\end{array}$ \\
\hline \multirow[t]{2}{*}{ LWS01 - Grating } & \multicolumn{2}{|c|}{$43.0-90.5$} & 0.29 & $150-300$ & $1000-2000$ & $\sim 80$ \\
\hline & \multicolumn{2}{|c|}{ 90.5-196.7 } & 0.60 & $150-300$ & $1000-2000$ & \\
\hline \multirow[t]{4}{*}{ LWS02 - Grating } & [O I] & 63.184 & 0.29 & 220 & 1400 & $81 \times 84^{\mathrm{a}}$ \\
\hline & {$[\mathrm{N}$ iI $]$} & 121.898 & 0.60 & 200 & 1500 & $78 \times 75^{\mathrm{a}}$ \\
\hline & {$[\mathrm{O} \mathrm{I}]$} & 145.525 & 0.60 & 240 & 1200 & $70 \times 65^{\mathrm{a}}$ \\
\hline & {$[\mathrm{C}$ II $]$} & 157.741 & 0.60 & 260 & 1100 & $71 \times 70^{\mathrm{a}}$ \\
\hline \multirow[t]{6}{*}{ LWS04-FP } & {$[\mathrm{O} \mathrm{I}]$} & 63.184 & 0.0078 & 8200 & 37 & $81 \times 84^{\mathrm{a}}$ \\
\hline & [Si I $]$ & 68.473 & 0.0084 & 8100 & 37 & " \\
\hline & {$[\mathrm{N}$ II $]$} & 121.898 & 0.0132 & 9300 & 32 & $78 \times 75^{\mathrm{a}}$ \\
\hline & [Si I $]$ & 129.682 & 0.0142 & 9300 & 33 & \\
\hline & [O I $]$ & 145.525 & 0.0163 & 9100 & 34 & $70 \times 65^{\mathrm{a}}$ \\
\hline & [C II] & 157.741 & 0.0175 & 9000 & 33 & $71 \times 70^{\mathrm{a}}$ \\
\hline \multirow[t]{6}{*}{ SWS02 - Grating } & {$[\mathrm{Fe} \mathrm{I}]$} & 24.042 & 0.018 & 1300 & 230 & $14 \times 27^{\mathrm{b}}$ \\
\hline & [S I $]$ & 25.249 & 0.018 & 1400 & 220 & "I \\
\hline & [Fe II] & 25.988 & 0.018 & 1400 & 220 & 11 \\
\hline & [Si II] & 34.815 & 0.022 & 1600 & 190 & $20 \times 33^{\mathrm{b}}$ \\
\hline & {$[\mathrm{Fe} \mathrm{I}]$} & 34.714 & 0.022 & 1600 & 190 & "I \\
\hline & [Fe II] & 35.349 & 0.022 & 1600 & 190 & 11 \\
\hline \multirow[t]{6}{*}{ SWS07 - FP } & {$[\mathrm{Fe} \mathrm{I}]$} & 24.042 & 0.00073 & 35000 & 9 & $10 \times 39^{b}$ \\
\hline & {$[\mathrm{S} \mathrm{I}]$} & 25.249 & 0.00073 & 35000 & 9 & "I \\
\hline & {$[\mathrm{Fe} I \mathrm{II}]$} & 25.988 & 0.00073 & 35000 & 9 & " \\
\hline & {$[\mathrm{Fe} \mathrm{I}]$} & 34.713 & 0.0012 & 30000 & 10 & $17 \times 40^{\mathrm{b}}$ \\
\hline & [Si II] & 34.815 & 0.0012 & 30000 & 10 & "I \\
\hline & [Fe II] & 35.349 & 0.0012 & 30000 & 10 & I \\
\hline
\end{tabular}

References: (a) Swinyard et al. 1998; (b) Leech et al. 2000.

$35 \mu \mathrm{m}$, [Si II], $35 \mu \mathrm{m}$, [O I] 63 and $146 \mu \mathrm{m}$, [Si I] 68 and $130 \mu \mathrm{m},[\mathrm{N} \mathrm{II}] 122 \mu \mathrm{m}$ and [C II] $158 \mu \mathrm{m}$. The [Ne II] $12.8 \mu \mathrm{m}$ line was observed from the ground with Irshell (see Appendix A). All the other line observations were made using the SWS (de Graauw et al. 1996) and the LWS (Clegg et al. 1996) on board ISO (Kessler et al. 1996).

\subsection{ISO observations}

Using the two spectrometers in both the grating and Fabry-Perot (FP) modes, we measured the atomic fine structure lines given in Table 1 . Columns 1 and 2 list the Astronomical Observation Template (AOT) modes used and the observed lines. Columns 3-6 state the instrumental resolution, the resolving power, the spectral resolutions and the beam size. Table 2 lists the sources in order of increasing central star temperature and gives the source positions, the AOTs used, the total ON or OFF integration time, and the date(s) of observation. OFF-source points were observed to determine the contribution from galactic cloud emission. The SWS grating, the SWS-FP, and the LWS grating typically consist of 24,3 and 56, respectively, scans per line. The number of scans forming the LWS-FP spectra varies from 3 to over 100 separate scans per line. The wavelength calibration of the SWS between 12 to $44.5 \mu \mathrm{m}$ is good to about $1 / 8$ of a resolution element for the grating and about $1 / 4$ of a resolution element for the FP. The absolute flux calibration is accurate to about $30 \%$. The wavelength calibration of the LWS is good to about $1 / 4$ of a resolution element for the grating and about a half of the resolution element for the FP. The absolute flux calibration for extended sources at $120 \mu \mathrm{m}$ is accurate to about $30 \%$ for sources $\lesssim 1000$ Jy. For bright ( $\gtrsim 1000 \mathrm{Jy})$ and weak ( $10-50 \mathrm{Jy})$ sources, the accuracy of the flux calibration drops to $50 \%$. Bright sources can cause some detectors to become non-linear and weak sources have calibration errors arising from uncertainties in the behavior of the detector dark current.

\subsection{ISO data reduction and line fluxes}

The data were reduced following standard reduction procedures. Initial data reduction was carried out using the Standard Processed Data (SPD) products from version 7.01 of the Off Line Processing (OLP). Post-pipeline reduction for our SWS data were performed using the Observer SWS Interactive Analysis package (OSIA) to derive the Auto Analysis Result (AAR). The calibration files from version 7.0 of the OLP were applied. Our SWS02 scans suffered from the presence of instrumental fringes. The residual fringes were removed to a large degree in OSIA, but low-level fringe artifacts still remain present in a few of our spectra. No lines were detected in any of the spectra tainted by fringes, although weak lines could 
Table 2. Observational parameters

\begin{tabular}{|c|c|c|c|c|c|c|c|}
\hline Source & Alternate Names & $\mathrm{OFF}$ & $\overline{\mathrm{RA}}(\mathrm{J} 2000)$ & DEC (J2000) & $\mathrm{AOT}$ & $T_{\text {int }}$ & Obs. Date \\
\hline IRC +10216 & $\begin{array}{l}\text { CW Leo } \\
\text { IRAS 09452+1330 }\end{array}$ & & $09: 47: 57.3$ & $13: 16: 42.8$ & LWS04 & 1852 & 28 may96 \\
\hline IRAS 15194-5115 & & & $15: 23: 05.0$ & $-51: 25: 59.1$ & $\begin{array}{l}\text { LWS01 } \\
\text { LWS02 }\end{array}$ & $\begin{array}{c}1612 \\
860\end{array}$ & $\begin{array}{l}14 \operatorname{mar} 97 \\
14 \operatorname{mar} 97\end{array}$ \\
\hline & & $\sqrt{ }$ & $15: 23: 05.0$ & $-51: 20: 59.0$ & $\begin{array}{l}\text { LWS02 } \\
\text { SWS02 }\end{array}$ & $\begin{array}{l}784 \\
970\end{array}$ & $\begin{array}{l}14 \operatorname{mar} 97 \\
05 \operatorname{aug} 97\end{array}$ \\
\hline LP And & $\begin{array}{l}\text { IRC }+40540 \\
\text { IRAS } 23320+4316\end{array}$ & & $23: 34: 27.7$ & $43: 33: 02.0$ & SWS07 & 2520 & $06 \operatorname{dec} 97$ \\
\hline IRAS $22272+5435$ & HD 235858 & & $22: 29: 10.3$ & $54: 51: 06.9$ & LWS04 & 1295 & 17nov96 \\
\hline & & $\sqrt{ }$ & $22: 29: 10.2$ & $54: 56: 06.9$ & $\begin{array}{l}\text { LWS04 } \\
\text { LWS04 }\end{array}$ & $\begin{array}{c}528 \\
2054\end{array}$ & $\begin{array}{l}17 \text { nov96 } \\
17 \text { may } 97\end{array}$ \\
\hline & & $\sqrt{ }$ & & & LWS04 & 1652 & 17 may 97 \\
\hline AC Her & IRAS $18281+2149$ & $\sqrt{ }$ & $\begin{array}{l}18: 30: 16.2 \\
18: 30: 16.2\end{array}$ & $\begin{array}{l}21: 52: 00.0 \\
21: 57: 00.0\end{array}$ & $\begin{array}{l}\text { LWS02 } \\
\text { LWS02 } \\
\text { SWS02 }\end{array}$ & $\begin{array}{l}812 \\
566 \\
970\end{array}$ & $\begin{array}{l}01 \mathrm{mar} 97 \\
01 \mathrm{mar} 97 \\
01 \mathrm{mar} 97\end{array}$ \\
\hline SAO 163075 & IRAS 19500-1709 & & $19: 52: 52.6$ & $-17: 01: 50.0$ & $\begin{array}{l}\text { LWS04 } \\
\text { LWS04 }\end{array}$ & $\begin{array}{l}2244 \\
2290\end{array}$ & $\begin{array}{l}26 \operatorname{apr} 97 \\
22 \operatorname{oct} 97\end{array}$ \\
\hline & & $\sqrt{ }$ & $19: 52: 52.5$ & $-16: 56: 49.6$ & $\begin{array}{l}\text { LWS04 } \\
\text { SWS02 } \\
\text { SWS02 }\end{array}$ & $\begin{array}{c}1012 \\
996 \\
994\end{array}$ & $\begin{array}{l}22 \operatorname{oct} 97 \\
26 \operatorname{apr} 97 \\
22 \operatorname{oct} 97\end{array}$ \\
\hline AFGL 2688 & Egg Nebula & $\sqrt{ }$ & $\begin{array}{l}21: 02: 18.8 \\
21: 02: 18.6\end{array}$ & $\begin{array}{l}36: 41: 37.8 \\
36: 46: 37.8\end{array}$ & $\begin{array}{l}\text { LWS04 } \\
\text { LWS04 } \\
\text { LWS04 } \\
\text { SWS07 }\end{array}$ & $\begin{array}{c}1080 \\
664 \\
3870 \\
6704\end{array}$ & $\begin{array}{l}\text { 05nov96 } \\
\text { 05nov96 } \\
\text { 21apr97 } \\
\text { 20oct96 }\end{array}$ \\
\hline Red Rectangle & $\begin{array}{l}\text { AFGL } 915 \\
\text { IRAS } 06176-1036 \\
\text { HD } 44179\end{array}$ & $\sqrt{ }$ & $\begin{array}{l}06: 19: 58.2 \\
06: 19: 58.2\end{array}$ & $\begin{array}{l}-10: 38: 14.0 \\
-10: 32: 14.0\end{array}$ & $\begin{array}{l}\text { LWS02 } \\
\text { LWS02 } \\
\text { SWS02 }\end{array}$ & $\begin{array}{l}854 \\
616 \\
970\end{array}$ & $\begin{array}{l}18 \text { oct } 97 \\
18 \text { oct } 97 \\
18 \text { oct } 97\end{array}$ \\
\hline IRAS $21282+5050$ & & $\sqrt{ }$ & $\begin{array}{l}21: 29: 58.4 \\
21: 29: 58.3\end{array}$ & $\begin{array}{l}51: 04: 00.8 \\
51: 09: 00.8\end{array}$ & $\begin{array}{l}\text { LWS04 } \\
\text { LWS04 } \\
\text { LWS04 } \\
\text { SWS07 }\end{array}$ & $\begin{array}{c}380 \\
528 \\
2158 \\
2384\end{array}$ & $\begin{array}{c}28 \text { oct } 96 \\
28 \text { oct } 96 \\
10 \text { may } 97 \\
28 \text { oct } 96\end{array}$ \\
\hline AFGL 618 & IRAS $04395+3601$ & & $04: 42: 53.6$ & $36: 06: 54.0$ & $\begin{array}{l}\text { LWS04 } \\
\text { SWS02 } \\
\text { SWS02 } \\
\text { SWS02 } \\
\text { SWS02 } \\
\text { SWS07 }\end{array}$ & $\begin{array}{c}11388 \\
672 \\
672 \\
672 \\
672 \\
1642\end{array}$ & $\begin{array}{l}\text { 11sep97 } \\
\text { 03oct97 } \\
\text { 03oct97 } \\
\text { 04oct97 } \\
04 \text { oct } 97 \\
\text { 03oct97 }\end{array}$ \\
\hline NGC 6720 & $\begin{array}{l}\text { Ring Nebula } \\
\text { M } 57 \\
\text { IRAS } 18517+3257\end{array}$ & $\sqrt{ }$ & $\begin{array}{l}18: 53: 32.2 \\
18: 53: 31.9\end{array}$ & $\begin{array}{l}33: 01: 34.2 \\
33: 11: 34.3\end{array}$ & $\begin{array}{l}\mathrm{LWS04} \\
\mathrm{LWS04} \\
\text { SWS02 }\end{array}$ & $\begin{array}{l}1276 \\
856 \\
1020\end{array}$ & $\begin{array}{l}\text { 16nov96 } \\
16 \text { nov96 } \\
16 \text { nov96 }\end{array}$ \\
\hline NGC 7027 & & $\sqrt{ }$ & $\begin{array}{l}21: 07: 01.5 \\
21: 07: 01.4\end{array}$ & $\begin{array}{l}42: 14: 10.3 \\
42: 19: 10.3\end{array}$ & $\begin{array}{l}\text { LWS04 } \\
\text { LWS04 } \\
\text { SWS07 } \\
\text { SWS07 }\end{array}$ & $\begin{array}{c}1290 \\
1366 \\
2934 \\
568\end{array}$ & $\begin{array}{c}\text { 29apr97 } \\
\text { 29apr97 } \\
\text { 20may96 } \\
\text { 21apr97 }\end{array}$ \\
\hline
\end{tabular}

have been masked by the fringe pattern. The SWS07 scans from 19-26 $\mu \mathrm{m}$ suffered a large number of cosmic ray hits resulting in numerous glitch tails. Since these FP observations contain only 3 scans per line and the glitch tails affect a significant part of the spectra, the glitch tails were fitted with an exponential and subtracted out. Post-pipeline reduction for our LWS data was performed using the LWS Interactive Analysis package (LIA). Spurious slopes in the spectra of the FP data were caused by grating positioning problems. The grating misalignment was corrected using LIA. Final processing such as the removal of bad data points, flat-fielding and averaging the scans were carried out in the ISO Spectral Analysis Package (ISAP).
Figure 1 shows the LWS02 grating spectrum of the [O I] $63 \mu \mathrm{m}$ line from the Red Rectangle. This line is among our weakest detections in the sample. Figure 2 shows all of our detected LWS- and SWS-FP lines and the ground based [Ne II] lines (see Sect. 3 for the line fit parameters). Our integrated line fluxes of detected lines and upper limits of undetected lines are given in Table 3. Column 4 lists either the observed line fluxes or the $3 \sigma$ upper limits. The rms values in Col. 5 were obtained after the spectra were smoothed to the instrumental resolution. The integrated line flux was acquired by fitting a Gaussian to the line profile (but see Sect. 3 for the FP line fitted fluxes). The uncertainty in the line fluxes are statistical, and the error 
Table 3. Observational results

\begin{tabular}{|c|c|c|c|c|c|}
\hline \multirow[t]{2}{*}{ Source } & \multirow[t]{2}{*}{$\mathrm{AOT}$} & \multicolumn{2}{|r|}{ Line } & $\begin{array}{c}\text { Flux } \\
10^{-13} \mathrm{erg}\end{array}$ & $\begin{array}{c}\mathrm{rms} \\
10^{-12} \mathrm{erg}\end{array}$ \\
\hline & & \multicolumn{2}{|c|}{$(\mu \mathrm{m})$} & $\mathrm{cm}^{2} \mathrm{~s}$ & $\overline{\mathrm{cm}^{2} \mathrm{~s} \mu \mathrm{m}}$ \\
\hline \multirow[t]{2}{*}{$\mathrm{IRC}+10216$} & \multirow[t]{2}{*}{ L04 } & $\overline{[\mathrm{O}} \mathrm{I}]$ & 63.184 & $<6$ & 20 \\
\hline & & {$[\mathrm{C}$ II $]$} & 157.741 & $<14$ & 30 \\
\hline IRAS 15194 & \multirow[t]{5}{*}{ S02 } & {$[\mathrm{Fe} \mathrm{I}]$} & 24.042 & $<5$ & 9 \\
\hline \multirow[t]{8}{*}{-5115} & & {$[\mathrm{~S} \mathrm{I}]$} & 25.249 & $<4$ & 8 \\
\hline & & {$[\mathrm{Fe} \quad \mathrm{II}]$} & 25.988 & $<2$ & 5 \\
\hline & & [Si II] & 34.815 & $<4$ & 6 \\
\hline & & {$[\mathrm{Fe}$ II $]$} & 35.349 & $<4$ & 6 \\
\hline & L01 & {$[\mathrm{C}$ II $]$} & 157.741 & $16 \pm 0.6^{\mathrm{a}}$ & 0.1 \\
\hline & \multirow[t]{3}{*}{ L02 } & {$[\mathrm{O}$ I $]$} & 63.184 & $<4$ & 0.5 \\
\hline & & $\mathrm{CO}$ & 145.518 & $1.8 \pm 0.5$ & 0.06 \\
\hline & & [C II $]$ & 157.741 & $12 \pm 0.5^{\mathrm{a}}$ & 0.07 \\
\hline \multirow[t]{2}{*}{ OFF } & \multirow[t]{2}{*}{ L02 } & {$[\mathrm{O} \mathrm{I}]$} & 63.184 & $<4$ & 0.4 \\
\hline & & {$[\mathrm{C}$ II $]$} & 157.741 & $10 \pm 0.6$ & 0.09 \\
\hline \multirow[t]{3}{*}{ LP And } & S07 & [Si II] & 34.815 & $<4$ & 100 \\
\hline & \multirow[t]{2}{*}{ L01 } & {$[\mathrm{O} \mathrm{I}]$} & 63.184 & $<30^{\mathrm{c}}$ & 10 \\
\hline & & {$[\mathrm{C} \mathrm{II}]$} & 157.741 & $<6^{\mathrm{c}}$ & 1 \\
\hline \multirow{4}{*}{$\begin{array}{r}\text { IRAS } 22272 \\
+5435\end{array}$} & - & {$[\mathrm{Ne} \mathrm{II]}$} & 12.814 & $<20$ & $=$ \\
\hline & \multirow{3}{*}{ L04 } & {$[\mathrm{O}$ I] } & 63.184 & $<60$ & 300 \\
\hline & & {$[\mathrm{O} \mathrm{I}]$} & 145.530 & $<10$ & 30 \\
\hline & & {$[\mathrm{C}$ II $]$} & 157.741 & $61 \pm 6^{\mathrm{a}}$ & 20 \\
\hline \multirow[t]{2}{*}{ OFF } & \multirow[t]{2}{*}{ L04 } & {$[\mathrm{O} \mathrm{I}]$} & 63.184 & $<30$ & 100 \\
\hline & & {$[\mathrm{C}$ II $]$} & 157.741 & $41 \pm 6$ & 20 \\
\hline \multirow[t]{9}{*}{ AC Her } & \multirow[t]{5}{*}{ S02 } & {$[\mathrm{Fe} \mathrm{I}]$} & 24.042 & $<0.9$ & 2 \\
\hline & & [S I] & 25.249 & $<0.9$ & 2 \\
\hline & & {$[\mathrm{Fe}$ II $]$} & 25.988 & $<1$ & 2 \\
\hline & & [Si II] & 34.815 & $<3$ & 5 \\
\hline & & {$[\mathrm{Fe}$ II $]$} & 35.349 & $<3$ & 4 \\
\hline & \multirow[t]{4}{*}{ L02 } & {$\left[\begin{array}{ll}\mathrm{O} & \mathrm{I}\end{array}\right]$} & 63.184 & $<4$ & 0.5 \\
\hline & & {$[\mathrm{N}$ II] } & 121.889 & $<0.9$ & 0.05 \\
\hline & & {$[\mathrm{O} \mathrm{I}]$} & 145.530 & $<0.3$ & 0.02 \\
\hline & & {$[\mathrm{C}$ II $]$} & 157.741 & $1.4 \pm 0.5^{\mathrm{a}}$ & 0.09 \\
\hline \multirow[t]{2}{*}{ OFF } & \multirow[t]{2}{*}{ L02 } & {$[\mathrm{O} \mathrm{I}]$} & 63.184 & $<7$ & 0.8 \\
\hline & & {$[\mathrm{C}$ II $]$} & 157.741 & $2.3 \pm 0.8$ & 0.08 \\
\hline \multirow[t]{9}{*}{$\mathrm{SAO} 163075$} & \multirow[t]{5}{*}{ S02 } & {$[\mathrm{Fe} \mathrm{I}]$} & 24.042 & $<1$ & 2 \\
\hline & & {$[\mathrm{S}$ I] } & 25.249 & $<2$ & 3 \\
\hline & & {$[\mathrm{Fe} \mathrm{II}]$} & 25.988 & $<1$ & 3 \\
\hline & & [Si II] & 34.815 & $<3$ & 4 \\
\hline & & {$[\mathrm{Fe}$ II $]$} & 35.349 & $<3$ & 4 \\
\hline & L01 & {$\left[\begin{array}{ll}\mathrm{O} & \mathrm{I}\end{array}\right]$} & 63.184 & $<10^{\mathrm{c}}$ & 4 \\
\hline & & {$[\mathrm{C}$ II $]$} & 157.741 & $<4^{\mathrm{c}}$ & 0.7 \\
\hline & L04 & {$[\mathrm{O}$ I] } & 63.184 & $<30$ & 200 \\
\hline & & [O I] & 145.530 & $<9$ & 20 \\
\hline OFF & L04 & {$[\mathrm{O}$ I] } & 63.184 & $<30$ & 200 \\
\hline
\end{tabular}

(a) ISM origin, (b) possible ISM origin, (c) ISO Data Archive and (d) ON-OFF from Liu et al. (2001).

depends on the method of fitting. For a Gaussian fit, the error in the flux was computed from the errors in the individual parameters such as the baseline level, the FWHM and the peak. The error given by the line fit was also checked by measuring the rms over the line free emission regions of the spectra and integrating over the full width of the line. Differences in the errors may arise from deviations in the behavior of the noise within the line emission
Table 3. continued

\begin{tabular}{|c|c|c|c|c|c|}
\hline \multirow[t]{2}{*}{ Source } & \multirow[t]{2}{*}{$\mathrm{AOT}$} & \multirow{2}{*}{\multicolumn{2}{|c|}{$\begin{array}{c}\text { Line } \\
(\mu \mathrm{m}) \\
\end{array}$}} & $\begin{array}{c}\text { Flux } \\
10^{-13} \mathrm{erg}\end{array}$ & $\begin{array}{c}\mathrm{rms} \\
10^{-12} \mathrm{erg}\end{array}$ \\
\hline & & & & $\mathrm{cm}^{2} \mathrm{~s}$ & $\overline{\mathrm{cm}^{2} \mathrm{~s} \mu \mathrm{m}}$ \\
\hline \multirow[t]{9}{*}{ AFGL 2688} & $\overline{-}$ & {$[\mathrm{Ne} \mathrm{II}]$} & 12.814 & $<60$ & - \\
\hline & \multirow[t]{2}{*}{ S07 } & [Fe II] & 25.988 & $<10$ & 600 \\
\hline & & [Si II] & 34.815 & $<20$ & 600 \\
\hline & \multirow[t]{6}{*}{ L04 } & {$[\mathrm{O} \mathrm{I}]$} & 63.184 & $<70$ & 300 \\
\hline & & [Si I] & 68.473 & $<70$ & 300 \\
\hline & & {$[\mathrm{N}$ iI $]$} & 121.889 & $<10$ & 40 \\
\hline & & [Si I ] & 129.682 & $<20$ & 50 \\
\hline & & {$[\mathrm{O}$ I] } & 145.530 & $<20$ & 50 \\
\hline & & {$[\mathrm{C}$ II $]$} & 157.741 & $<30$ & 50 \\
\hline \multirow[t]{4}{*}{ OFF } & \multirow[t]{4}{*}{ L04 } & {$[\mathrm{O} \mathrm{I}]$} & 63.184 & $<60$ & 200 \\
\hline & & {$[\mathrm{N}$ iI $]$} & 121.889 & $<9$ & 20 \\
\hline & & {$[\mathrm{O} \mathrm{I}]$} & 145.530 & $<10$ & 30 \\
\hline & & {$[\mathrm{C}$ II $]$} & 157.741 & $<40$ & 80 \\
\hline \multirow[t]{11}{*}{ Red Rectangle } & - & {$[\mathrm{Ne} I \mathrm{II}]$} & 12.814 & $<20$ & - \\
\hline & \multirow[t]{5}{*}{ S02 } & {$[\mathrm{Fe} \mathrm{I}]$} & 24.042 & $<2$ & 4 \\
\hline & & {$[\mathrm{S} \mathrm{I}]$} & 25.249 & $<2$ & 3 \\
\hline & & {$[\mathrm{Fe} \quad \mathrm{II}]$} & 25.988 & $<3$ & 5 \\
\hline & & {$[\mathrm{Si}$ II] } & 34.815 & $<4$ & 6 \\
\hline & & {$\left[\begin{array}{ll}\mathrm{Fe} & \mathrm{II}\end{array}\right]$} & 35.349 & $<2$ & 3 \\
\hline & L01 & {$\left[\begin{array}{ll}\mathrm{O} & \mathrm{I}\end{array}\right]$} & 63.184 & $28 \pm 4^{\mathrm{d}}$ & - \\
\hline & \multirow[t]{4}{*}{ L02 } & {$[\mathrm{O}$ I $]$} & 63.184 & $20 \pm 5$ & 2 \\
\hline & & {$[\mathrm{N}$ iI $]$} & 121.889 & $<1$ & 0.08 \\
\hline & & {$[\mathrm{O}$ I $]$} & 145.530 & $<0.3$ & 0.02 \\
\hline & & {$[\mathrm{C}$ II $]$} & 157.741 & $5.3 \pm 0.6^{\mathrm{a}}$ & 0.1 \\
\hline \multirow[t]{2}{*}{$\mathrm{OFF}$} & \multirow[t]{2}{*}{ L02 } & {$[\mathrm{O} \mathrm{I}]$} & 63.184 & $<3$ & 0.3 \\
\hline & & {$[\mathrm{C}$ II $]$} & 157.741 & $4.0 \pm 0.6$ & 0.02 \\
\hline \multirow{10}{*}{$\begin{array}{r}\text { IRAS } 21282 \\
+5050\end{array}$} & - & {$[\mathrm{Ne} \mathrm{II}]$} & 12.814 & $43 \pm 2$ & - \\
\hline & \multirow[t]{3}{*}{ S07 } & {$[\mathrm{S} \mathrm{I}]$} & 25.249 & $<10$ & 600 \\
\hline & & [Fe II] & 25.988 & $<20$ & 900 \\
\hline & & [Si II] & 34.815 & $<10$ & 400 \\
\hline & \multirow[t]{3}{*}{ L01 } & {$\left[\begin{array}{ll}\mathrm{O} & \mathrm{I}\end{array}\right]$} & 63.184 & $290 \pm 10^{\mathrm{d}}$ & - \\
\hline & & {$[\mathrm{O}$ I] } & 145.530 & $13 \pm 1^{\mathrm{d}}$ & - \\
\hline & & {$[\mathrm{C}$ II $]$} & 157.741 & $34 \pm 2^{\mathrm{d}}$ & - \\
\hline & \multirow[t]{3}{*}{ L04 } & {$[\mathrm{O}$ I] } & 63.184 & $410 \pm 60$ & 300 \\
\hline & & [O I] & 145.530 & $<7$ & 10 \\
\hline & & {$[\mathrm{C}$ II $]$} & 157.741 & $85 \pm 6$ & 10 \\
\hline \multirow[t]{2}{*}{ OFF } & \multirow[t]{2}{*}{ L04 } & {$[\mathrm{O} \mathrm{I}]$} & 63.184 & $<80$ & 300 \\
\hline & & [C II $]$ & 157.741 & $<30$ & 60 \\
\hline
\end{tabular}

(a) ISM origin, (b) possible ISM origin, (c) ISO Data Archive and (d) ON-OFF from Liu et al. (2001).

regions. If they differ significantly, then the errors derived independently from the rms were used.

\subsection{ISM contamination}

For the lines where both the $\mathrm{ON}$ and $\mathrm{OFF}$ positions had detections, we consider only those observations where the ON scan was at least two times higher than the OFF scan to be associated with the source. We followed this conservative approach because our confidence to distinguish a detection was limited by confusion with background emission. Without a line map of the region, 
Table 3. continued

\begin{tabular}{|c|c|c|c|c|c|}
\hline Source & $\mathrm{AOT}$ & \multicolumn{2}{|c|}{$(\mu \mathrm{m})$} & $\begin{array}{c}\begin{array}{c}\text { Flux } \\
10^{-13} \mathrm{erg}\end{array} \\
\mathrm{cm}^{2} \mathrm{~s}\end{array}$ & $\begin{array}{c}\text { rms } \\
\frac{10^{-12} \mathrm{erg}}{\mathrm{cm}^{2} \mathrm{~s} \mu \mathrm{m}}\end{array}$ \\
\hline \multirow[t]{11}{*}{ AFGL 618} & - & {$[\mathrm{Ne} \mathrm{II}]$} & 12.814 & $140 \pm 20$ & $-r$ \\
\hline & \multirow[t]{3}{*}{ S02 } & [S I $]$ & 25.249 & $<7$ & 10 \\
\hline & & {$[\mathrm{Fe} \mathrm{II}]$} & 25.988 & $<6$ & 10 \\
\hline & & [Si II] & 34.815 & $<9$ & 10 \\
\hline & S07 & [Si II] & 34.815 & $<7$ & 200 \\
\hline & \multirow[t]{2}{*}{ L01 } & {$[\mathrm{O} \mathrm{I}]$} & 63.184 & $100^{\mathrm{e}}$ & - \\
\hline & & {$[\mathrm{C}$ II $]$} & 157.741 & $10^{\mathrm{b}, \mathrm{e}}$ & - \\
\hline & \multirow[t]{4}{*}{ L04 } & {$[\mathrm{O} \mathrm{I}]$} & 63.184 & $160 \pm 20$ & 100 \\
\hline & & {$[\mathrm{N}$ II $]$} & 121.889 & $<6$ & 20 \\
\hline & & {$[\mathrm{O} \mathrm{I}]$} & 145.530 & $<4$ & 8 \\
\hline & & {$[\mathrm{C}$ II $]$} & 157.741 & $<10$ & 30 \\
\hline \multirow[t]{13}{*}{ NGC 6720} & \multirow[t]{5}{*}{ S02 } & {$[\mathrm{Fe} \mathrm{I}]$} & 24.042 & $<0.4$ & 0.8 \\
\hline & & {$[\mathrm{S}$ I $]$} & 25.249 & $<0.5$ & 0.9 \\
\hline & & [O IV] & 25.890 & $15 \pm 0.4^{\mathrm{f}}$ & 2 \\
\hline & & [Si II] & 34.815 & $12 \pm 1$ & 7 \\
\hline & & [Fe II] & 35.349 & $<3$ & 5 \\
\hline & \multirow[t]{4}{*}{ L01 } & {$[\mathrm{O}$ I] } & 63.184 & $581 \pm 8^{\mathrm{d}}$ & - \\
\hline & & {$[\mathrm{N}$ II $]$} & 121.889 & $39 \pm 2^{\mathrm{d}}$ & - \\
\hline & & [O I] & 145.530 & $18 \pm 1^{\mathrm{d}}$ & - \\
\hline & & [C II] & 157.741 & $68 \pm 2^{\mathrm{d}}$ & - \\
\hline & \multirow[t]{4}{*}{ L04 } & {$[\mathrm{O} \mathrm{I}]$} & 63.184 & $440 \pm 50$ & 500 \\
\hline & & {$[\mathrm{N}$ II $]$} & 121.889 & $28 \pm 9$ & 20 \\
\hline & & {$[\mathrm{O} \mathrm{I}]$} & 145.530 & $<30$ & 60 \\
\hline & & [C II $]$ & 157.741 & $41 \pm 6$ & 30 \\
\hline \multirow[t]{2}{*}{ OFF } & \multirow[t]{2}{*}{ L04 } & {$[\mathrm{O} \mathrm{I}]$} & 63.184 & $<50$ & 200 \\
\hline & & {$[\mathrm{C}$ II $]$} & 157.741 & $<10$ & 20 \\
\hline \multirow[t]{17}{*}{ NGC 7027} & - & {$[\mathrm{Ne} I \mathrm{II}]$} & 12.814 & $1000 \pm 100$ & - \\
\hline & \multirow[t]{6}{*}{$\mathrm{S} 07$} & {$[\mathrm{Fe} \mathrm{I}]$} & 24.042 & $<10$ & 600 \\
\hline & & {$[\mathrm{S}$ I $]$} & 25.249 & $<20$ & 900 \\
\hline & & [Fe II] & 25.988 & $<10$ & 600 \\
\hline & & {$[\mathrm{Fe} \mathrm{I}]$} & 34.714 & $<30$ & 700 \\
\hline & & [Si II] & 34.815 & $100 \pm 20$ & 800 \\
\hline & & [Fe II] & 35.349 & $<30$ & 700 \\
\hline & \multirow[t]{4}{*}{ L01 } & {$\left[\begin{array}{ll}\mathrm{O} & \mathrm{I}\end{array}\right]$} & 63.184 & $6060 \pm 170^{\mathrm{d}}$ & - \\
\hline & & {$[\mathrm{N}$ II $]$} & 121.889 & $4.9 \pm 0.5^{\mathrm{d}}$ & - \\
\hline & & {$[\mathrm{O} \mathrm{I}]$} & 145.530 & $213 \pm 3^{\mathrm{d}}$ & - \\
\hline & & {$[\mathrm{C}$ II $]$} & 157.741 & $426 \pm 5^{\mathrm{d}}$ & - \\
\hline & \multirow[t]{6}{*}{ L04 } & {$[\mathrm{O} \mathrm{I}]$} & 63.184 & $6200 \pm 200$ & 400 \\
\hline & & {$[\mathrm{Si}$ I $]$} & 68.473 & $<80$ & 300 \\
\hline & & {$[\mathrm{N}$ II $]$} & 121.889 & $<20$ & 50 \\
\hline & & [Si I ] & 129.682 & $<10$ & 30 \\
\hline & & {$[\mathrm{O}$ I $]$} & 145.530 & $130 \pm 9$ & 40 \\
\hline & & [C II] & 157.741 & $350 \pm 20$ & 50 \\
\hline \multirow[t]{3}{*}{$\mathrm{OFF}$} & \multirow[t]{3}{*}{ L04 } & {$[\mathrm{O} \mathrm{I}]$} & 63.184 & $<80$ & 400 \\
\hline & & [Si I ] & 129.682 & $<7$ & 20 \\
\hline & & [C II] & 157.741 & $<30$ & 60 \\
\hline
\end{tabular}

(a) ISM origin, (b) possible ISM origin, (c) ISO Data Archive, (d) ON-OFF from Liu et al. (2001), (e)

Justtanont (private com.) and (f) possibly HeII 25.900.

it was difficult to assess the point-to-point background variations. The lines most likely originating from interstellar gas are noted in Table 3. OFF source [O I] contamination was negligible in all the C-rich sources, whereas background [CII] emission was found in IRAS 151945115, IRAS 22272+5435, ACHer, Red Rectangle and
IRAS 21282+5050. The LWS01 grating detection of [C II] in AFGL 618 listed in Table 3, originally reported by Herpin \& Cernicharo (2000), may possibly originate from the ISM and not from AFGL 618 as claimed by Herpin \& Cernicharo (2000). The problem with this measurement is that no OFF position was taken for AFGL 618, so we cannot know for certain whether the [C II] is from the ISM or AFGL 618. However, the flux from the OFF positions of our other sources range from 4-60 $10^{-13} \mathrm{erg} \mathrm{cm}^{-2} \mathrm{~s}^{-1}$. This range includes the $1010^{-13} \mathrm{erg} \mathrm{cm}^{-2} \mathrm{~s}^{-1}$ [C II] flux detected for AFGL 618 and thus, casts doubt on its association with AFGL 618. If we had detected the [C II] line in our LWS04 FP observation, then we would have had a line profile and systemic velocity that would aid in its identification with AFGL 618 or the ISM. Unfortunately, the $[\mathrm{C}$ II] line flux measured in the LWS01 grating is below our sensitivity for the FP observation. This issue may be resolved with future airborne observations.

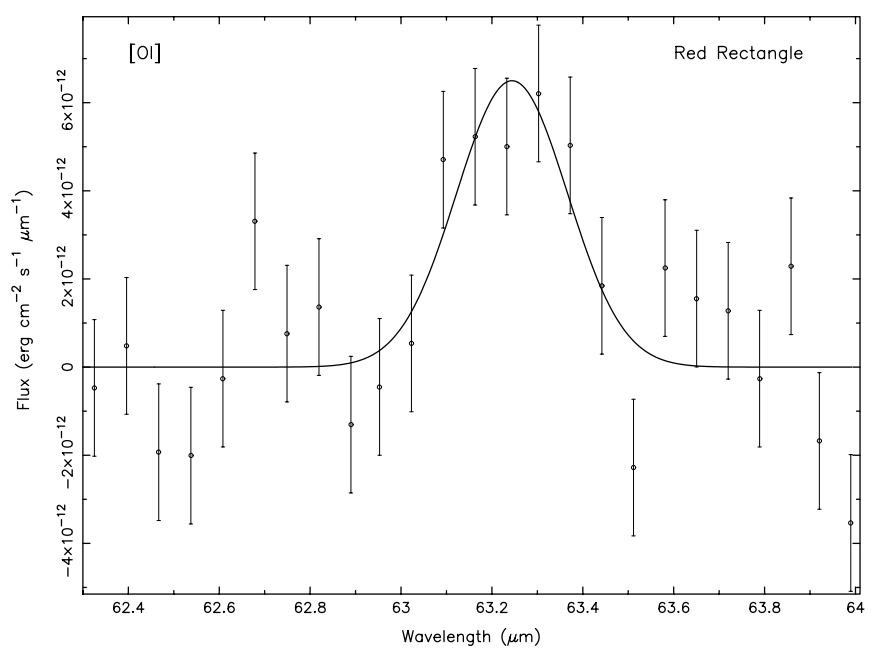

Fig. 1. The Red Rectangle: LWS02 grating spectrum of the [O I] $63 \mu \mathrm{m}$ line. This line is representative of the weakest grating detection in our sample. The Red Rectangle is the coolest star with detectable FIR line emission (see Sect. 4 of text)

\subsection{Detections and non-detections}

Five of the twelve C-rich evolved stars have been detected in one or more of the FIR atomic lines: the Red Rectangle, IRAS 21282+5050, AFGL 618, NGC 6720 and NGC 7027. The planetary nebula, NGC 7027, has the most lines detected and has the brightest line fluxes. The protoplanetary nebula, the Red Rectangle, is the weakest source detected. The [O I] $63 \mu \mathrm{m}$ line is always the brightest line of those detected. The LWS01 grating line fluxes for the Red Rectangle, IRAS 21282+5050, NGC 6720 and NGC 7027 from Liu et al. (1997, 2001), and AFGL 618 from Justtanont (private communication) are also listed in Table 3 for comparison with our LWS04 FP data. In general, the FP line fluxes are lower than the corresponding grating fluxes. Our more accurate line fits for the FP data 
result in higher line fluxes than reported in Table 3 because the ISAP Gaussian fits do not include the line wings and thus underestimate the total line flux (see Sect. 3 and Table 4). Because the grating data have better flux calibration, we use the grating line fluxes in our comparison with model calculations (see Sect. 5.2).

FIR line emission was not detected in the remaining seven objects in our C-rich sample: IRC +10216 , IRAS 15194-5115, LP And, IRAS 22272+5435, AC Her, SAO 163075 and AFGL 2688. For these non-detected sources, our LWS-FP data was found to be consistent with the LWS01 grating observations available from the ISO data archive. The archive search also yielded new [O I] $63 \mu \mathrm{m}$ and [C II] $158 \mu \mathrm{m}$ upper limits for LP And and SAO 163075 (PI: Barlow).

\section{Fabry-Perot line profile analysis}

The FP lines provide the important kinematic information for our investigation. Hence, we have modeled the line profiles to extract expansion velocities $(V)$, line center velocities $\left(V_{\mathrm{LSR}}\right)$ and more accurate line fluxes. The instrumental profiles of the LWS and SWS FP's follow Airy functions, which have notable line wings. For two reasons it is very important to take into account these line wings when modeling the line. First, we want to determine if the observed line wings are real or instrumental. If the line wings are real, they could indicate a shockedgas component to the line emission. Second, the emission from line wings need to be included in the total line flux. Since ISAP provides only Gaussian fits, we developed our own FP line fitting program to derive the expansion velocities and total line fluxes. Below we describe the line fitting procedure and discuss the derived kinematics in the context of PDRs versus shocks.

\subsection{Line profile fits}

Models of the spectral line profiles were derived from the ISO data and the ground based [Ne II] data by using a program we developed in the software package, Mathematica 4.0. The basic algorithm convolves a model line profile for the source with the instrumental spectral profile. The convolved model is sampled to match the ISO spectral sampling and is then compared with the observed data to determine the goodness of fit. The expansion velocity, the peak flux level, and the line center of the input model are varied over a reasonable parameter space. Chisquared minimization was used to find the model parameters which best fit the ISO data. The line fitting errors were determined by finding the region of parameter space where $\Delta \chi^{2} \leq 3.5$, the $68 \%$ confidence interval for 3 free parameters (Press et al. 1995 and references therein). This confidence ellipsoid was then projected onto the individual parameter axes and the endpoints of the projection were used to conservatively estimate the errors. The goodness of our noise estimate $(\sigma)$ was also checked by comparing the minimum $\chi^{2}$ value with the number of degrees of freedom. All the lines agrees well with this test except for the NGC 7027 [O I] $63 \mu \mathrm{m}$ line, our strongest line, where systematic errors are probably dominant. The best fit expansion velocity $(V)$, line center, peak flux and the total integrated fluxes are listed in Table 4 . The spectra and the convolved fits of the FP detections and the [Ne II] lines are shown in Fig. 2.

We used an Airy function for the instrumental profile of both the LWS-FP and SWS-FP. For the LWS-FP, the instrumental resolution as a function of wavelength was provided by Bruce Swinyard (private communication) and was approximately $35 \mathrm{~km} \mathrm{~s}^{-1}$. For the SWS-FP, Feuchtgruber (1998) found that the spectral resolutions are consistent with the values given in the SWS Observers Manual and are approximately $10 \mathrm{~km} \mathrm{~s}^{-1}$. For the ground based [Ne II] observations, we used a Gaussian function for the instrumental profile with a FWHM of approximately $33 \mathrm{~km} \mathrm{~s}^{-1}$.

We tried three different input source line profiles for the ISO LWS and SWS data: a parabola, a flat-top parabola (described by Knapp \& Morris 1985) and a Gaussian. The convolved line profiles for each of the three different input source line profiles were indistinguishable for most lines because the majority of lines were only marginally resolved by the FP. Since the true line shape could not be constrained, for consistency we chose to report the fitting parameters derived by using a flat-topped parabolic input model for all the ISO LWS and SWS line fits. This choice is justified from the molecular CO line profiles, which for most of the sources are consistent with flat-topped parabolas. We chose to give line widths at the HWHM points as opposed to the HWZP points because the signal to noise ratio of the data are better at the HWHM. Flat-top parabolic profiles have steep sides, thus differences between the HWHM and HWZP points are only $\sim 15 \%$. For the ground based [NeII] spectra we tried only the Gaussian source line profile. For AFGL 618 the fit only covered the core emission because the [Ne II] line is situated near a $\mathrm{C}_{2} \mathrm{H}_{2}$ absorption feature.

To show that our convolved model gives a better fit to the ISO data than the ISAP Gaussian, we take the case of NGC 7027. Figure 3 shows the NGC 7027 [O I] $63 \mu \mathrm{m}$ spectrum fitted with both the ISAP Gaussian and the convolved model. The ISAP Gaussian fit can falsely suggest the presence of real line wings. Our convolved model demonstrates that the apparent line wings are instrumental in nature. In Table 4, the expansion velocities and integrated fluxes derived from the ISAP Gaussian fit are listed in Cols. 7 and 8 for comparison with the convolved model fits. The ISAP expansion velocity errors include only the statistical errors of the fit. The errors in the convolved model also include the effects of deconvolving the instrumental profile. For the detected atomic fine structure lines at NGC 7027, all the model line widths and line centers agree with the ${ }^{12} \mathrm{CO}$ line parameters. In contrast, the line widths derived from the ISAP Gaussian fit are revealed to be overestimates, with exception to [Si II] 

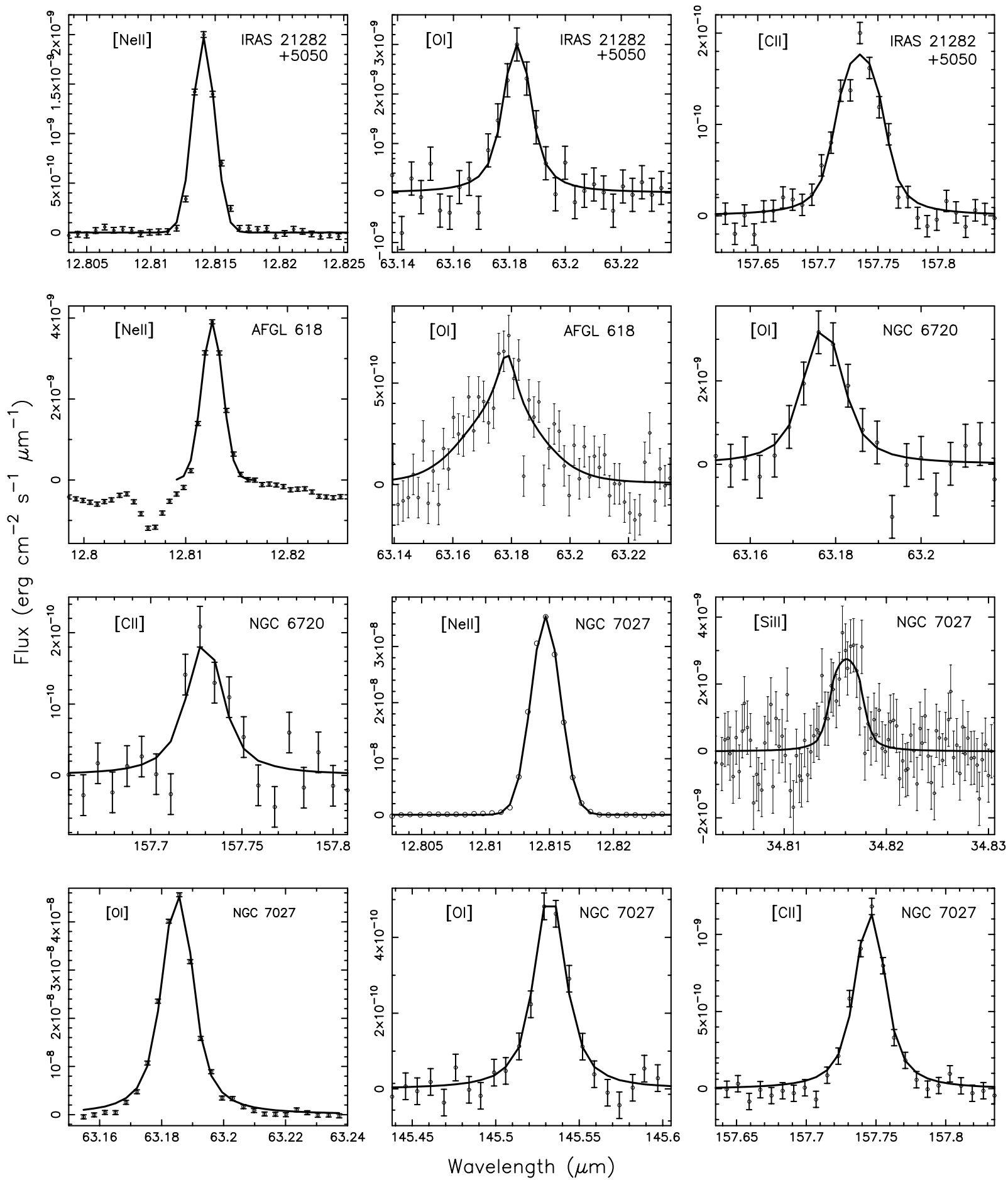

Fig. 2. ISO FP spectra and Irshell spectra with convolved fits

$35 \mu \mathrm{m}$ where the line has been resolved by the SWS-FP. The integrated fluxes derived from the ISAP Gaussian fit are also found to be slightly under-determined because of the less accurate fits to the observed data.

\subsection{Kinematics of the low-excitation atomic gas}

The fitted model line parameters can be used to interpret whether the atomic lines originate from PDRs or through shocks. One method compares the observed line fluxes with the theoretically predicted line fluxes from PDR and shock models (discussed in Sects. 5.2 and 6, respectively). A second method uses the kinematic information extracted from the observed line profiles to ascertain their origins. PDRs lie at interfaces between ionized and molecular gas, so we would expect the FIR cooling lines associated with circumstellar PDRs to have outflow velocities comparable to the ionized and molecular gas expansion velocities derived from the [Ne II] $12.8 \mu \mathrm{m}$ line 
Table 4. Line fitting parameters

\begin{tabular}{|c|c|c|c|c|c|c|c|c|c|}
\hline \multirow[b]{2}{*}{ Source } & \multirow[b]{2}{*}{ Line } & \multicolumn{4}{|c|}{ Convolved Model Line Fit } & \multicolumn{2}{|c|}{ ISAP Gaussian Fit } & \multicolumn{2}{|c|}{${ }^{12} \mathrm{CO}$ Line Parameters } \\
\hline & & $\begin{array}{c}V \\
(\mathrm{HWHM}) \\
\left(\mathrm{km} \mathrm{s}^{-1}\right)\end{array}$ & $\begin{array}{c}\text { Line Center } \\
\left(V_{\mathrm{LSR}}\right) \\
\left(\mathrm{km} \mathrm{s}^{-1}\right)\end{array}$ & $\begin{array}{l}\text { Peak Flux } \\
\frac{10^{-11} \mathrm{erg}}{\mathrm{cm}^{2} \mathrm{~s} \mu \mathrm{m}}\end{array}$ & $\begin{array}{c}\text { Total Flux } \\
\frac{10^{-13} \mathrm{erg}}{\mathrm{cm}^{2} \mathrm{~s}}\end{array}$ & $\begin{array}{c}V \\
(\mathrm{HWHM}) \\
\left(\mathrm{km} \mathrm{s}^{-1}\right) \\
\end{array}$ & $\begin{array}{l}\text { Total Flux } \\
\frac{10^{-13} \mathrm{erg}}{\mathrm{cm}^{2} \mathrm{~s}}\end{array}$ & $\begin{array}{c}V_{\exp } \\
(\mathrm{HWZP}) \\
\left(\mathrm{km} \mathrm{s}^{-1}\right)\end{array}$ & $\begin{array}{c}V_{\mathrm{LSR}} \\
\left(\mathrm{km} \mathrm{s}^{-1}\right)\end{array}$ \\
\hline $\begin{array}{l}\text { IRAS } 22272+5435 \\
\quad(\mathrm{OFF})\end{array}$ & {$[\mathrm{C}$ II $] 158$} & $\begin{array}{c}9_{-9}^{+7} \\
15_{-15}^{+17}\end{array}$ & $\begin{array}{l}-48 \pm 4 \\
-43 \pm 8\end{array}$ & $\begin{array}{l}25 \pm 2 \\
14 \pm 4\end{array}$ & $\begin{array}{l}73 \pm 19 \\
47 \pm 36\end{array}$ & $\begin{array}{l}22 \pm 1 \\
28 \pm 1\end{array}$ & $\begin{array}{l}61 \pm 6 \\
41 \pm 6\end{array}$ & 10 & -28 \\
\hline IRAS $21282+5050$ & $\begin{array}{l}{[\mathrm{Ne} \text { II }] 12.8} \\
{[\mathrm{O} \text { I } 63} \\
{\left[\mathrm{C}_{\mathrm{II}}\right] 158}\end{array}$ & $\begin{array}{c}17 \pm 2 \\
22 \pm 10 \\
41 \pm 5\end{array}$ & $\begin{array}{c}13 \pm 1 \\
10 \pm 5 \\
3 \pm 5\end{array}$ & $\begin{array}{c}196 \pm 2 \\
300 \pm 30 \\
18 \pm 1\end{array}$ & $\begin{array}{c}43 \pm 3 \\
470 \pm 160 \\
93 \pm 15\end{array}$ & $\begin{array}{l}- \\
32 \pm 1 \\
42 \pm 1\end{array}$ & $\begin{array}{c}- \\
410 \pm 60 \\
85 \pm 6\end{array}$ & 16 & 14 \\
\hline $\begin{array}{l}\text { AFGL } 618 \\
\text { model } 1 \\
\text { model } 2 \\
\text { model } 3(\mathrm{~b}) \\
\text { model } 3(\mathrm{n})\end{array}$ & $\begin{array}{l}{[\mathrm{Ne} \text { II] }] 12.8} \\
{[\mathrm{O} \text { I }] 63} \\
{[\mathrm{O} \text { I] } 63} \\
{[\mathrm{O} \text { I] } 63} \\
{[\mathrm{O} \text { I] } 63}\end{array}$ & $\begin{array}{c}23 \pm 1 \\
20 \\
68 \pm 15 \\
66 \pm 22 \\
4_{-4}^{+12}\end{array}$ & $\begin{array}{l}-22 \pm 1 \\
-21 \\
-29 \pm 8 \\
-24 \pm 5 \\
-21 \pm 5\end{array}$ & $\begin{array}{c}390 \pm 10 \\
73 \\
48 \pm 10 \\
12_{-12}^{+18} \\
52_{-18}^{+12}\end{array}$ & $\begin{array}{c}100 \pm 3 \\
120 \\
150 \pm 60 \\
160 \pm 40(\mathrm{~b}+\mathrm{n})\end{array}$ & $\begin{array}{l}- \\
- \\
65 \pm 3 \\
- \\
-\end{array}$ & $\begin{array}{c}- \\
- \\
160 \pm 20 \\
- \\
-\end{array}$ & $\begin{array}{c}190(J=3-2) \\
19(J=3-2)\end{array}$ & $\begin{array}{l}-19 \\
-22\end{array}$ \\
\hline NGC 6720 & $\begin{array}{l}{[\mathrm{O} \text { I }] 63} \\
{[\mathrm{C} \text { II] }] 158}\end{array}$ & $\begin{array}{l}20_{-20}^{+14} \\
15_{-15}^{+10}\end{array}$ & $\begin{array}{l}-10 \pm 6 \\
-6 \pm 5\end{array}$ & $\begin{array}{c}320 \pm 50 \\
18 \pm 6\end{array}$ & $\begin{aligned} 480 & \pm 300 \\
59 & \pm 43\end{aligned}$ & $\begin{array}{l}36 \pm 1 \\
20 \pm 1\end{array}$ & $\begin{aligned} 440 & \pm 50 \\
41 & \pm 6\end{aligned}$ & 27 & -1 \\
\hline NGC 7027 & $\begin{array}{l}{[\mathrm{Ne} \text { II }] 12.8} \\
{[\mathrm{Si} \text { II] }] 34.8} \\
{[\mathrm{O} \text { I] } 63} \\
{[\mathrm{O} \text { I }] 145} \\
{[\mathrm{C} \text { II }] 158}\end{array}$ & $\begin{array}{l}27 \pm 1 \\
16 \pm 6 \\
23 \pm 1 \\
15 \pm 8 \\
19 \pm 5\end{array}$ & $\begin{array}{l}27 \pm 1 \\
25 \pm 4 \\
22 \pm 3 \\
28 \pm 4 \\
22 \pm 3\end{array}$ & $\begin{aligned} 3520 & \pm 10 \\
280 & \pm 80 \\
4500 & \pm 100 \\
49 & \pm 3 \\
120 & \pm 10\end{aligned}$ & $\begin{array}{c}1020 \pm 30 \\
120 \pm 80 \\
7000 \pm 200 \\
154 \pm 31 \\
400 \pm 80\end{array}$ & $\begin{array}{c}- \\
15 \pm 1 \\
31.3 \pm 0.3 \\
26 \pm 1 \\
27 \pm 1\end{array}$ & $\begin{array}{c}- \\
100 \pm 20 \\
6200 \pm 200 \\
130 \pm 9 \\
350 \pm 20\end{array}$ & 20 & 26 \\
\hline
\end{tabular}

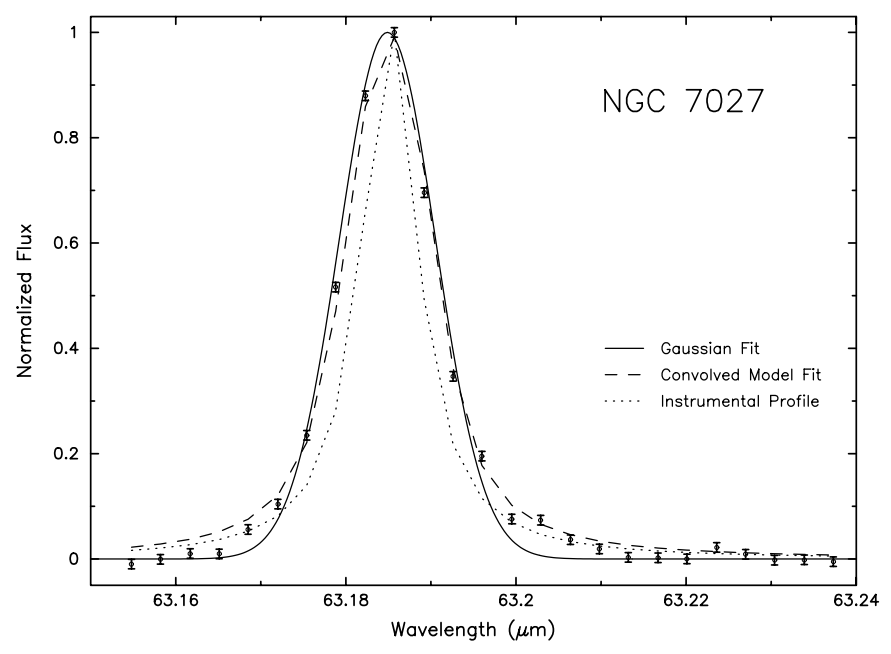

Fig. 3. NGC 7027: Comparison of the ISAP Gaussian fit (solid), convolved model fit (dashed) and the instrumental profile (dotted). The line wings are instrumental

and the ${ }^{12} \mathrm{CO}$ lines, respectively. This holds true for the atomic fine structure lines detected in IRAS $21282+5050$, NGC 6720 and NGC 7027. Shocks can develop when the fast post-AGB wind encounters the slower AGB wind ejected during the previous epoch of mass loss. The wind driven shocks can compress, heat, accelerate and dissociate the molecular gas. Since the shocked emission occurs in the post-shock, accelerated gas, the line widths may be significantly broadened and the line centers may be shifted appreciably from the velocity flow of the ambient molecular gas (HM89). The observed line kinematics suggest the presence of shocks in AFGL 618.
Detailed discussions of the individual objects are given below. Since the LWS-FP and SWS-FP wavelength calibrations are $\sim 10 \mathrm{~km} \mathrm{~s}^{-1}$ and $\sim 3 \mathrm{~km} \mathrm{~s}^{-1}$, respectively, we consider $V_{\text {LSR }}$ velocity discrepancies greater than the errors in the wavelength calibration to be significant in the line center comparisons.

IRAS 22272+5435 - The [C II] $158 \mu \mathrm{m}$ line very likely originates from the ISM because of three points: 1) [C II] was detected at the OFF position with over $60 \%$ of the $\mathrm{ON}$ source integrated flux, 2) the very narrow [C II] line seen at the ON position matches the shape of the instrumental profile and 3) the line centers for both the ON and OFF positions are $\sim 20 \mathrm{~km} \mathrm{~s}^{-1}$ offset from the ${ }^{12} \mathrm{CO} V_{\mathrm{LSR}}$. The large uncertainty in the model outflow velocity resulted from the poor signal-to-noise of the data and the fact that the line is unresolved.

IRAS $21282+5050$ - The [O I] $63 \mu \mathrm{m}$ line is consistent with the ${ }^{12} \mathrm{CO}$ and the [Ne II] line parameters, suggesting a PDR origin for the lines in this source. The [C II] $158 \mu \mathrm{m}$ line is about twice as broad and is offset by $11 \mathrm{~km} \mathrm{~s}^{-1}$ from the ${ }^{12} \mathrm{CO} V_{\mathrm{LSR}}$. Since IRAS $21282+5050$ is located in the plane of the galaxy (Galactic coordinates: $93.99,-0.12)$, interstellar emission may have contaminated the line. Although [C II] was not detected at the selected FP OFF position, background [C II] was detected by Liu et al. (2001) from grating observations.

AFGL 618 - The [Ne II] line has a comparable $V_{\mathrm{HWHM}}$ to that measured for ${ }^{12} \mathrm{CO} J=1-0$ suggesting that the ionized gas is expanding with a velocity similar to that of the molecular gas. However, the ${ }^{12} \mathrm{CO}$ transistions also suggest a broad velocity component with outflow velocities $>190 \mathrm{~km} \mathrm{~s}^{-1}$ (Gammie et al. 1989; Cernicharo et al. 1989). 

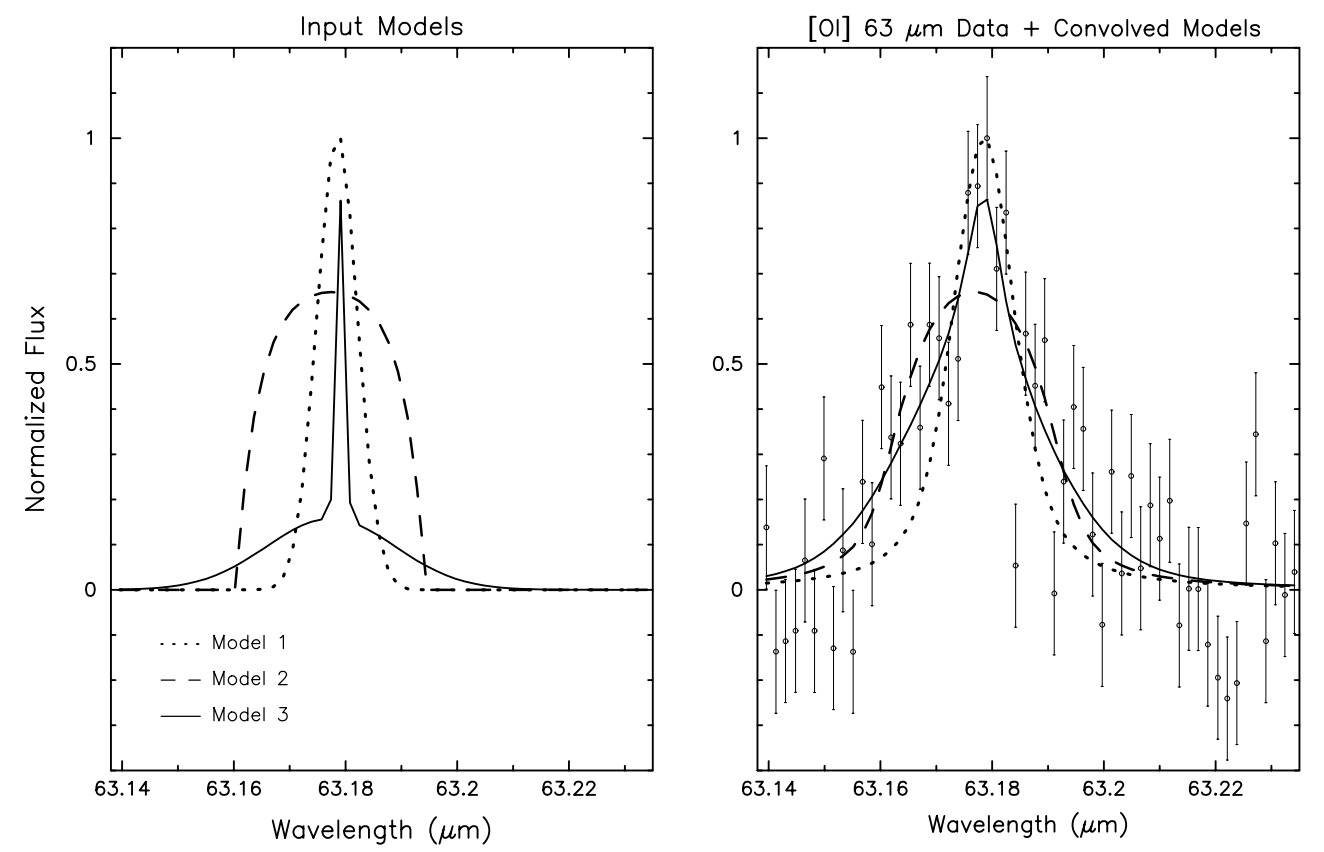

Fig. 4. AFGL 618: Comparison of model 1 - single component Gaussian (dotted), model 2 - flat-topped parabolic (dashed) and model 3 - double component Gaussian model fit (solid)

In addition, there is a tentative detection of line wings on the core of the $[\mathrm{CI}$ ] line (Young 1997). Interestingly, the [O I] $63 \mu \mathrm{m}$ line appears much broader than the [Ne II] line. Figure 4 shows the results of three models for the [O I] $63 \mu \mathrm{m}$ line. The first model, a single component Gaussian with a $V_{\mathrm{HWHM}}$ of $20 \mathrm{~km} \mathrm{~s}^{-1}$ to approximate the main ${ }^{12} \mathrm{CO}$ line profile, fits the center of the observed spectrum but lacks the line wings. The second model, our best-fit, single component parabola with a HWHM of $68 \pm 15 \mathrm{~km} \mathrm{~s}^{-1}$, adequately fits the line wings but not the central feature. The third model has two components: a narrow core feature and broad extended wings. This model is composed of two Gaussians of varying widths, peaks fluxes and velocity offsets. Gaussians were chosen because the broad ${ }^{12} \mathrm{CO}$ line wings appears to match that profile. The two Gaussians were then added together to form a composite profile prior to the convolution. The best fit was again found by searching parameter space for the minimum $\chi^{2}$. The broad component has an outflow velocity (HWHM) of $66 \pm 22 \mathrm{~km} \mathrm{~s}^{-1}$ and the narrow component has an expansion velocity of $4_{-4}^{+12}$. The large uncertainty of the narrow feature can be understood by the fact that it is unresolved by the LWS-FP.

Although the kinematics of the narrow feature are not well constrained, the considerable difference in expansion velocity between the narrow and broad components is analogous to ${ }^{12} \mathrm{CO}$. The peak flux of the [O I] $63 \mu \mathrm{m}$ narrow feature is about 8 times the peak of the broad component. The same flux comparison can be made for various transitions of ${ }^{12} \mathrm{CO}$. The narrow to broad peak flux ratio for the $J=3-2,2-1$ and $1-0$ transition of ${ }^{12} \mathrm{CO}$ are 7.2 , 13 and 19, respectively (Gammie et al. 1989; Cernicharo et al. 1989; Meixner et al. 1998). The relative strength of the broad component to the narrow component appears to be greater in the higher ${ }^{12} \mathrm{CO}$ transitions, possibly due to shock excitation (Justtanont et al. 2000). For the [O I] $63 \mu \mathrm{m}$ line, the broad component is much stronger than the narrow component in comparison to the $[\mathrm{CI}]$ line or the ${ }^{12} \mathrm{CO}$ lines. This suggests that the [O I] line is more dominated by the shock component than the other lines. The two component model appears generally similar to the line shape seen in ${ }^{12} \mathrm{CO}(J=3-2)$, but with the velocity outflow cutoff at $66 \mathrm{~km} \mathrm{~s}^{-1}$. The $66 \mathrm{kms}$ [O I] outflow velocity is comparable to the bipolar medium velocity wind mapped in ${ }^{12} \mathrm{CO} J=1-0$ (Meixner et al. 1998).

NGC 6720 - Due to the large extent of NGC 6720 (130" across) and the limited size of the ISO LWS beam $\left(70-80^{\prime \prime}\right)$, the observations were pointed $10^{\prime \prime} \mathrm{S}$ and $47^{\prime \prime} \mathrm{W}$ off source center, toward the ${ }^{12} \mathrm{CO}(J=2-1)$ peak (Bachiller et al. 1989). The ${ }^{12} \mathrm{CO}(J=2-1)$ line profile at that position clearly does not resemble a flat-topped parabola because an offset piece of NGC 6720 was observed. Due to the poor signal-to-noise of the data and the resultant large uncertainty in the velocity outflow, a more detailed model was not warranted. Nevertheless, the similarities between the [O I], [C II], [C I] (Bachiller et al. 1994) and ${ }^{12} \mathrm{CO}$ lines argues for a PDR origin in this source.

NGC 7027 - The line parameters for the [Si II] $34.8 \mu \mathrm{m},[\mathrm{O} \mathrm{I}] 63$ and $145 \mu \mathrm{m}$ and the [C II] $158 \mu \mathrm{m}$ lines show comparable expansion velocities to the ionized gas, as traced by [Ne II] $12.8 \mu \mathrm{m}$, and the molecular gas, as traced in ${ }^{12} \mathrm{CO}$. Hence, these lines arise in a PDR for this source. Low-level broad line wings have been detected in several CO rotational transitions (e.g. Jamminet et al. 1991) and in the [C I] line (Young et al. 1999) in this source and may indicate a small component of shocked gas. Our FP observations would not be sensitive to faint line wings because of the instrumental profile. Nevertheless, this 


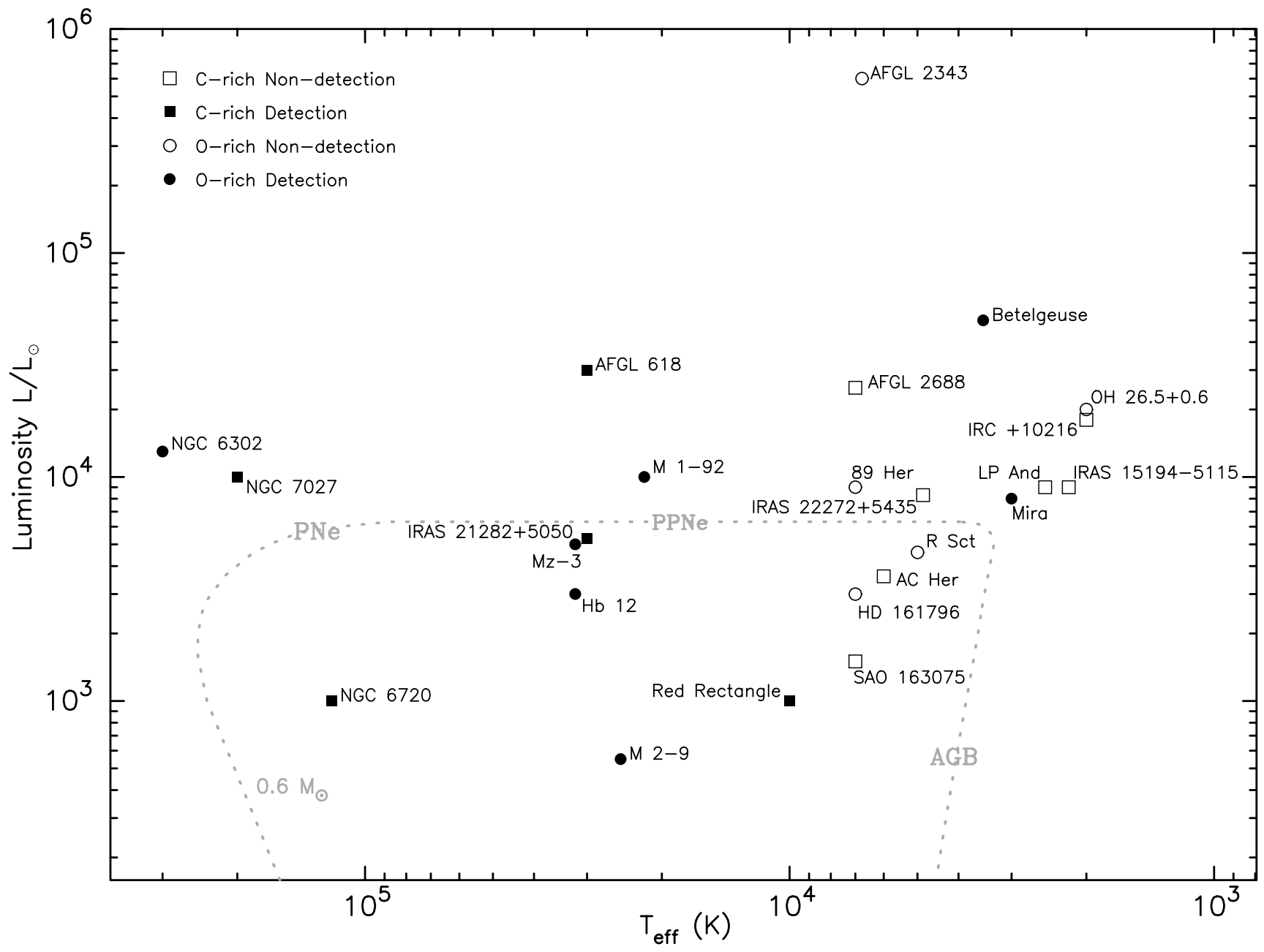

Fig. 5. The observed source distribution plotted in a H-R diagram

possible shocked gas component contribution to our detected lines must be minor.

\section{Dependence of the atomic line emission on the stellar parameters}

The HR diagram (Fig. 5) graphically displays the evolutionary distribution of our evolved star sample for both C-rich and O-rich sources. The latter are taken from Paper II. In Table 5 we list the spectral type, stellar temperature, distance and luminosity for each of the C-rich sources in the plot. Only 12 out of the 24 objects had FIR line detections: all 3 PNe, 4 young PNe/PPNe, 3 PPNe, 1 red supergiant star and 1 AGB star. There appears to be no preferential chemical type where FIR lines were seen the detections contain both C-rich and O-rich sources.

The HR diagram shows a clear temperature boundary between where atomic cooling lines were detected and not detected. The Red Rectangle apparently lies at the critical $T_{\text {eff }}(\sim 10000 \mathrm{~K})$ where FIR lines first emerges. The Red Rectangle, a known spectroscopic binary (Van Winckel et al. 1995), has HeI recombination emission that corresponds to a central star $T_{\text {eff }}>20000 \mathrm{~K}$.
However, the combined photospheres of the binary system appear cooler $(\sim 10000 \mathrm{~K})$ suggesting that this emission is produced by chromospheric or coronal excitation (e.g. Kelly \& Latter 1995). Below 10 000 K, atomic fine structure lines were not detected in any of the sources except for two unusual O-rich sources: the red supergiant Betelgeuse and the AGB star Mira which has a hot binary companion (discussed in Paper II). Five O- and C-rich sources located about $3000 \mathrm{~K}$ below this temperature divide, with a luminosity range of 3 orders of magnitude, do not show FIR line emission. This further suggests that the $T_{\text {eff }}$ is the deciding factor and not the luminosity. Moreover, the absence of FIR lines below $\sim 10000 \mathrm{~K}$ shows that the PDR created by the ISRF on the external surface of the circumstellar molecular envelope does not contribute to these FIR lines (see Sect. 5.4). Nebular characteristics such as morphology, chemistry, shocks and total mass do not seem to play a role. For instance, the PPNe AFGL 2688 and AFGL 618 both show conspicuous axial symmetry, C-rich chemistry, the presence of shocks, a massive molecular envelope $\left(\sim 1 M_{\odot}\right.$; Table 7$)$ but FIR lines were only observed in AFGL 618 because it is located above the critical $T_{\text {eff }}$. Finally, the distances are not systematically different for 
Table 5. Sample of C-rich nebulae

\begin{tabular}{|c|c|c|c|c|c|}
\hline Source & Type & $\begin{array}{c}\text { Spectral } \\
\text { Type }\end{array}$ & $\begin{array}{c}\text { Temperature } \\
(\mathrm{K})\end{array}$ & $\begin{array}{c}D \\
(\mathrm{kpc})\end{array}$ & $\begin{array}{c}L \\
\left(10^{3} L_{\odot}\right)\end{array}$ \\
\hline $\mathrm{IRC}+10216$ & AGB & $\mathrm{C} 9^{\mathrm{S}, \mathrm{a}}$ & $2000^{\mathrm{b}}$ & $0.15^{\mathrm{b}, \mathrm{c}}$ & $18^{\mathrm{b}, \mathrm{c}}$ \\
\hline IRAS $15194-5115$ & AGB & $\mathrm{C}^{\mathrm{d}}$ & $2200^{\mathrm{e}}$ & $0.6^{\mathrm{d}, \mathrm{e}, \mathrm{f}}$ & $9^{\mathrm{d}, \mathrm{e}, \mathrm{f}}$ \\
\hline LP And & AGB & $\mathrm{C} 8^{\mathrm{S}, \mathrm{a}}$ & $2500^{\mathrm{f}}$ & $0.7^{\mathrm{f}, \mathrm{g}}$ & $9^{\mathrm{f}}$ \\
\hline IRAS $22272+5435$ & $\mathrm{PPN}$ & $\mathrm{G} \mathrm{Ia}^{\mathrm{h}}$ & $4850^{\mathrm{h}}$ & $1.7^{i}$ & $8.3^{\mathrm{i}}$ \\
\hline AC Her & $\mathrm{PPN}$ & $\mathrm{F} 4 \mathrm{Ib}^{\mathrm{S}, \mathrm{a}}$ & $6000^{\mathrm{j}, \mathrm{k}}$ & $1.43^{\mathrm{H}}$ & $3.6^{\mathrm{H}, 1}$ \\
\hline SAO 163075 & $\mathrm{PPN}$ & $\mathrm{F} 2-6^{\mathrm{m}}$ & $7000^{\mathrm{m}}$ & $1^{\mathrm{n}}$ & $1.5^{\mathrm{n}}$ \\
\hline AFGL 2688 & $\mathrm{PPN}$ & F5 Iae ${ }^{\circ}$ & $7000^{\circ}$ & $1.2^{\mathrm{p}}$ & $25^{\mathrm{p}, \mathrm{o}}$ \\
\hline Red Rectangle & PPN & $\mathrm{A} 0^{\mathrm{q}}$ & $10000^{\mathrm{q}}$ & $0.38^{\mathrm{H}}$ & $1^{1}$ \\
\hline IRAS $21282+5050$ & PPN/Young PN & O9-9.5 & $30000^{\mathrm{r}, \mathrm{s}}$ & $3^{\mathrm{s}}$ & $5.3^{\mathrm{s}}$ \\
\hline AFGL 618 & PPN/Young PN & $\mathrm{B}^{\circ}$ & $30000^{\circ}$ & $1.7^{\mathrm{t}}$ & $30^{\mathrm{n}}$ \\
\hline NGC 6720 & $\mathrm{PN}$ & pec. $^{u}$ & $120000^{\mathrm{u}}$ & $0.92^{\mathrm{u}}$ & $1^{\mathrm{u}}$ \\
\hline NGC 7027 & $\mathrm{PN}$ & pec. ${ }^{\mathrm{v}}$ & $198000^{v}$ & $1^{\mathrm{w}}$ & $10^{\mathrm{x}}$ \\
\hline
\end{tabular}

References: (S) Simbad; (H) Hipparcos; (a) Kholopov et al. 1985; (b) Groenewegen 1997; (c) Groenewegen et al. 1998a; Bagnulo et al. 1995; (d) Ryde et al. 1999; (e) Le Bertre 1997; (f) Groenewegen et al. 1998b; (g) Cohen \& Hitchon 1996; (h) Hrivnak 1995; (i) Szczerba et al. 1997; (j) Shenton et al. 1992; (k) Giridhar et al. 1998; (l) Alcolea \& Bujarrabal 1991; (m) Hrivnak et al. 1989; (n) Bujarrabal et al. 1992; (o) Calvet \& Cohen 1978; (p) Skinner et al. 1997; (q) Kelly \& Latter 1995; Knapp et al. 1995; (r) Crowther et al. 1998; (s) Meixner et al. 1997; (t) Bujarrabal et al. 1988; (u) Cahn 1984; (v) Latter et al. 2000; (w) Jourdain de Muizon et al. 1990; Graham et al. 1993; (x) Liu et al. 1997.

the sources with $T_{\text {eff }}$ above and below $10000 \mathrm{~K}$, thus the beam dilution is not inclined to be higher for the sources with non-detections.

Above this $T_{\text {eff }}$ boundary of $\sim 10000 \mathrm{~K}$, the number of detectable lines increase with higher $T_{\text {eff }}$. Furthermore, the intensity of the FIR line emission also increases with rising $T_{\text {eff }}$. These trends are expected for PDRs because with increasing $T_{\text {eff }}$, more UV photons are available for photodissociation and photoelectric heating, which increases the intensity of the atomic cooling lines. The picture presented by the HR diagram consistently supports the case for PDRs as the excitation mechanism for these lines.

\section{PDR models}

Having established that the atomic cooling lines most likely originate from PDRs, we can proceed to compare the observed line intensities with the emergent intensities predicted from the theoretical PDR models. Most PDR models found in the literature (e.g. TH85) have been developed for the interstellar medium which has a higher abundance of oxygen compared to carbon. Here we use a new type of PDR model created specifically for the enhanced levels of carbon relative to oxygen found in the C-rich circumstellar environments of our sample. This carbon enrichment changes the chemistry of the PDR, requiring the inclusion of chemical reactions involving species such as $\mathrm{C}_{2}$ and $\mathrm{C}_{2} \mathrm{H}_{2}$. It also modifies the energetics because the photoelectric heating mechanism, which dominates the heating of a PDR, is enhanced by the presence of small carbon grains and polycyclic aromatic hydrocarbons (PAHs). Below we briefly summarize some aspects of the model, noting that the full blown model will be described in a forth coming paper (Latter \& Tielens in preparation). Following this description, we present a comparison of our data with the models.

\subsection{PDR code}

The PDR code models the chemistry, energetics and radiative transfer of a plane-parallel, semi-infinite slab. The far-ultraviolet (FUV) photons, which are attenuated primarily by the dust, drive the heating and the chemistry of the neutral gas layer. The main heating mechanisms are FUV pumping of $\mathrm{H}_{2}$ and photoelectric ejection of electrons from primarily PAHs and small dust grains (TH85; Hollenbach \& Tielens 1997). The PDR code uses the photoelectric heating rates derived by Bakes \& Tielens (1994; hereafter BT94) which includes an MRN grain size distribution (proportional to $a^{-3.5}$, where $a$ is the grain radius; Mathis et al. 1977) that extends down to the PAH domain. The gas is cooled through atomic fine structure lines such as [O I] $63 \mu \mathrm{m}$ and [C II] $158 \mu \mathrm{m}$, rovibrational transitions of $\mathrm{H}_{2}$, and by rotational lines of molecules such as $\mathrm{CO}, \mathrm{HCO}^{+}$and $\mathrm{HCN}$. The chemistry includes C-rich molecules (e.g. $\mathrm{C}_{2}$ and $\mathrm{C}_{2} \mathrm{H}_{2}$ ) not found in the ISM models, and a limited PAH chemistry that uses the $\mathrm{PAH}^{-}$, $\mathrm{PAH}$ and $\mathrm{PAH}^{+}$species. The chemical network includes 1270 reactions between 94 species using the elements: $\mathrm{H}$, $\mathrm{He}, \mathrm{C}, \mathrm{N}, \mathrm{O}, \mathrm{Si}, \mathrm{S}, \mathrm{Mg}$ and Fe. The $\mathrm{H}_{2}$ formation on grains is as discussed by TH85 and Sternberg \& Dalgarno (1995). The PDR code calculates the energy balance 
Table 6. Standard model parameters

\begin{tabular}{lc}
\hline Parameters & Value \\
\hline Carbon abundance & $1.510^{-3}$ \\
Oxygen abundance & $5.010^{-4}$ \\
Nitrogen abundance & $210^{-4}$ \\
Silicon abundance & $3.510^{-6}$ \\
Sulfur abundance & $7.910^{-6}$ \\
Magnesium abundance & $1.310^{-6}$ \\
Iron abundance & $2.510^{-7}$ \\
PAH abundance & $n(\mathrm{PAH}) / n=10^{-7}$ \\
Gas density $\left(\mathrm{cm}^{-3}\right)$ & $100<n<10^{6}$ \\
Incident FUV flux $\left(G_{0}\right)$ & $10<G<10^{8}$ \\
Turbulent Doppler velocity $\left(\mathrm{km} \mathrm{s}^{-1}\right)$ & 0.75 \\
FUV dust absorption/visual extinction & $1.8($ at $2000 \AA)$ \\
\hline
\end{tabular}

(the heating and cooling rates and the gas and dust temperatures) and the chemical composition (abundances and the column densities) as a function of optical depth into the neutral gas. The emergent intensities of the atomic and molecular cooling lines are then computed.

Table 6 summarizes the model input parameters. The two main input parameters are the gas density, $n$, and the incident FUV flux, $G$, in units of $G_{0}$, the average value for the ISRF, $\left(G_{0}=1.610^{-3} \mathrm{ergs} \mathrm{cm}^{-2} \mathrm{~s}^{-1}\right.$; Habing 1968). We assume that $G$ has the same spectral shape as the interstellar radiation field (Draine 1978) which is approximately that of a $T_{\text {eff }}=30000 \mathrm{~K}$ blackbody in the spectral range of interest (cf. Spaans et al. 1994). The turbulent velocities are expected to be small in these envelopes so we adopt a value of $0.75 \mathrm{~km} \mathrm{~s}^{-1}$. We have opted to use only one set of abundances rather than source specific abundances because we are conducting a general comparison of PDR theory and observations. Except for carbon and nitrogen, standard ISM elemental abundances found in TH85 and Steenberg \& Shull (1988) were used (Table 6). For carbon, we adopted an abundance of $1.510^{-3}$. This value is the average of the carbon abundances for six of our sources: IRAS $22272+5435\left(5.610^{-3}\right.$; Zacs et al. 1995), AC Her (1.62 10 ${ }^{-4}$; Giridhar et al. 1998), SAO 163075 (9.1 10 Van Winckel \& Reyniers 2000), Red Rectangle ${ }^{1}$ (4.6 10 ${ }^{-4}$; Waelkens et al. 1992), NGC6720 (6.5 10 ${ }^{-4}$; Kwitter \& Henry 1998) and NGC 7027 (1.3 10 ${ }^{-3}$; Middlemass 1990). For nitrogen we adopted an abundance of $210^{-4}$. This value is an average of the nitrogen abundances for four

\footnotetext{
${ }^{1}$ While most of these evolved stars began as O-rich AGB stars and, via third dredge-up, became C-rich (e.g. Iben 1983), the Red Rectangle actually shows evidence of its O-rich past with the detection of crystalline silicates in its circum-binary disk (Waters et al. 1998). Despite the presence of this O-rich dust, the Red Rectangle is still considered C-rich due to the presence of PAH emission and the strong [C I $609 \mu \mathrm{m}$ line emission (Knapp et al. 2000).
}

of our sources: SAO 163075 (2.8 $10^{-4}$; Van Winckel \& Reyniers 2000), Red Rectangle (8.9 10 1992), NGC 6720 (2.5 10 ${ }^{-4}$; Kwitter \& Henry 1998) and NGC 7027 (1.9 10-4; Middlemass 1990).

\subsubsection{An example model}

We present some details for a model with $G=10^{5} G_{0}$ and $n=10^{5} \mathrm{~cm}^{-3}$ in order to illustrate the model results and how they differ from standard ISM models (e.g. TH85) which have been used to model the results in Paper II. Figure 6 shows the heating, cooling and the relevant chemical abundance structure of the PDR as a function of $A_{V}$. One of the main differences between our C-rich models and the ISM PDR models is that the abundance of carbon is always higher than the abundance of oxygen. At the inner surface of the PDR, oxygen is in neutral atomic form $(\mathrm{O})$ while the carbon is singly ionized $\left(\mathrm{C}^{+}\right)$. At an $A_{V} \sim 3$, oxygen and carbon combine to form $\mathrm{CO}$ through many competing reactions which leads to the complex abundance structure. Nearly all the oxygen becomes incorporated into $\mathrm{CO}$, but only a fraction of the carbon becomes depleted. Most of the remaining carbon recombines to form neutral atomic carbon (C). Note that the abundance of $\mathrm{C}$ is higher than $\mathrm{CO}$ from an $A_{V} \sim 3$ to 8 . Beyond an $A_{V} \sim 8$, carbon combines to form carbon-bearing molecules such as $\mathrm{C}_{2}$ and acetylene $\left(\mathrm{C}_{2} \mathrm{H}_{2}, A_{V}>10\right)$. In the ISM PDR models (see e.g. Fig. 9 of TH85), the carbon and oxygen play the reverse roles. Due to the higher oxygen abundance, carbon is depleted in the formation of CO.

Figure 7 shows the gas and dust temperature as a function of $A_{V}$. The peak gas temperature in our C-rich PDR models is approximately three times higher than that in standard ISM PDR models. The reasons behind this temperature increase are related to the increase in carbon abundance. Firstly, PAHs are more abundant in C-rich evolved stars than the ISM. Recent PDR models have shown the importance of photoelectric heating from PAHs and small dust grains on the neutral atomic interstellar gas (e.g. Kaufman et al. 1999, BT94). In the ISM, many studies indicate that the dust is composed largely of a mixture of carboneous and silicate grains (e.g. MRN 1977). The grain composition found in circumstellar environments are more chemically homogeneous: silicate grains are detected in O-rich objects whereas carboneous grains are found in C-rich sources. Thus the photoelectric heating will depend heavily on the photoelectric properties of the grains formed in their respective environments. C-rich sources have shown ample evidence for PAHs (e.g. Cohen et al. 1989; Cohen 1986; Aitken \& Roche 1982), hence these sources can be well modeled by the photoelectric heating from small carbon grains and PAHs using the heating formalism in BT94. Secondly, the enhanced C abundance will increase the photoelectric heating through a three fold process: 1) the higher neutral $\mathrm{C}$ ionization will 

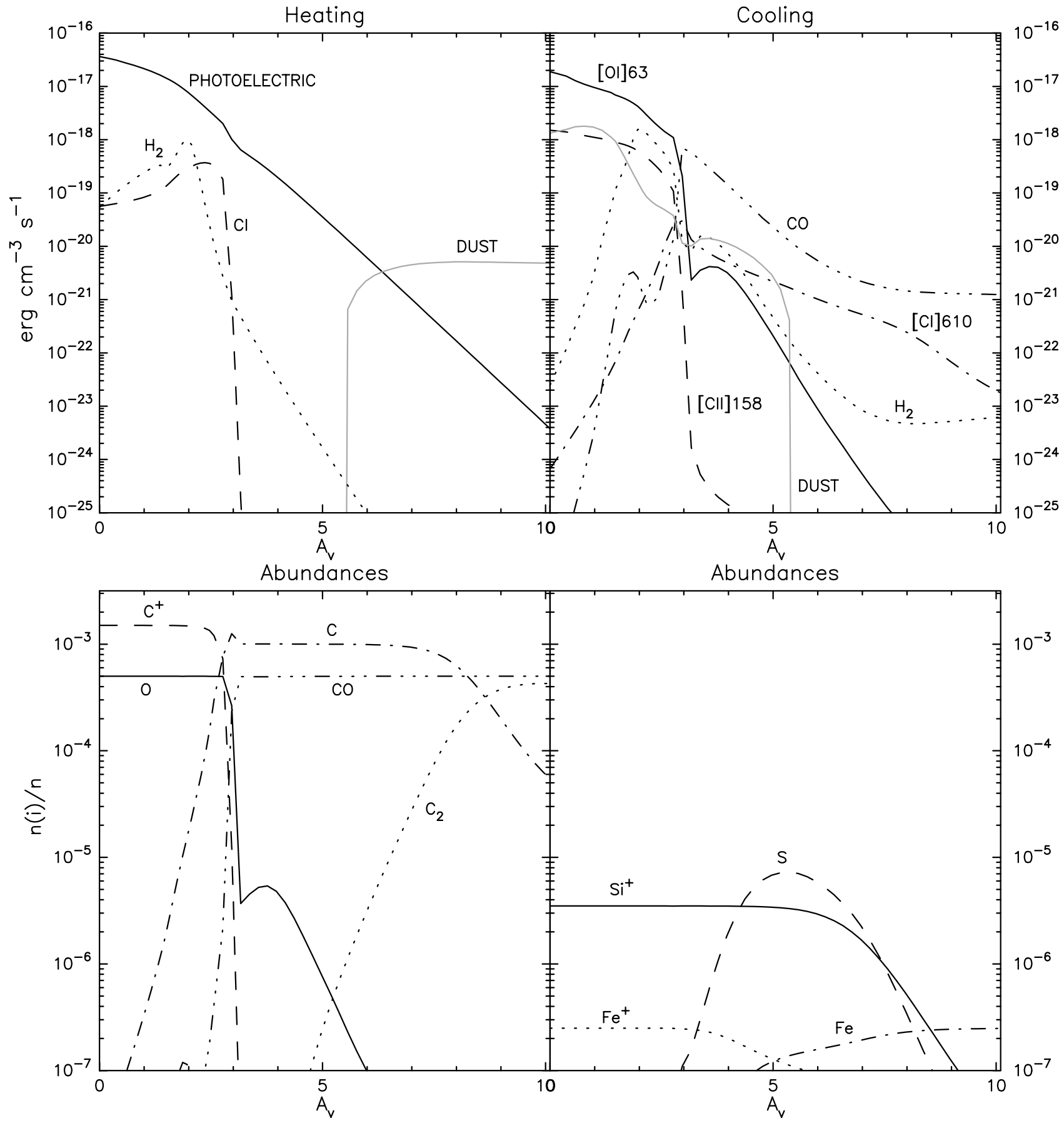

Fig. 6. Structure of the PDR for $G=10^{5} G_{0}$ and $n=10^{5} \mathrm{~cm}^{-3}$. The upper two panels show the heating and the cooling rates. The bottom two panels show the chemical abundances as a function of nebular depth

increase the electron density, 2) the higher electron density will lower the grain charge and 3 ) the lower ionization potential of the grain will increase the photoelectric yield.

\subsection{Comparison of observations with PDR line emission theory}

PDR model calculations for a broad range of $G=$ $10-10^{8} G_{0}$ and $n=100-10^{6} \mathrm{~cm}^{-3}$ are presented for nine atomic cooling lines: [O I] 63 and $146 \mu \mathrm{m},[\mathrm{C}$ II] $158 \mu \mathrm{m}$, [Si II] $35 \mu \mathrm{m}$, [Fe II] 26 and $35 \mu \mathrm{m}$, [Fe I] 24 and $35 \mu \mathrm{m}$ and
[C I] $609 \mu \mathrm{m}$ (Fig. 8). The series of curves for the neutral and ionized lines, show the predicted intensities over the $G$ and $n$ parameter space.

The line intensity data are plotted against the incident FUV flux. $G$ was derived from stellar parameters such as the luminosity, the fraction of far-UV photons emitted by the central star and the inner radius of the PDR (Tables 5 and 7) using a method described in Paper II. The largest uncertainty in deriving $G$ is the angular, inner PDR radius (see Table 7 for references). An image of a PDR gas tracer such as vibrationally excited $\mathrm{H}_{2} 2.12 \mu \mathrm{m}$, which peaks 
Table 7. Parameters used for the comparison of our observations with PDR models and derived densities and masses

\begin{tabular}{lcccccccccc}
\hline Source & $\begin{array}{c}\theta \\
\left({ }^{\prime \prime}\right)\end{array}$ & $\begin{array}{c}R_{i} \\
(\mathrm{~cm})\end{array}$ & $\begin{array}{c}\left(4 \pi \theta^{2}\right)^{-1} \\
\left(\mathrm{sr}^{-1}\right)\end{array}$ & $F_{\mathrm{UV}}$ & $\begin{array}{c}G \\
\left(G_{0}\right)\end{array}$ & $\begin{array}{c}n \\
\left(\mathrm{~cm}^{-3}\right)\end{array}$ & $\begin{array}{c}F_{\mathrm{c}} \\
M_{\text {ionized }} \\
\left(M_{\odot}\right)\end{array}$ & $\begin{array}{c}M_{\text {atomic }} \\
\left(M_{\odot}\right)\end{array}$ & $\begin{array}{c}M_{\text {molecular }} \\
\left(M_{\odot}\right)\end{array}$ \\
\hline IRC +10216 & $0.13^{\mathrm{a}}$ & $310^{14}$ & $210^{11}$ & $5.310^{-12}$ & 0.21 & - & - & - & - & - \\
IRAS 15194-5115 & $0.06^{\mathrm{b}}$ & $510^{14}$ & $10^{12}$ & $9.610^{-11}$ & 0.66 & - & - & - & - & - \\
LP And & $0.05^{\mathrm{b}}$ & $510^{14}$ & $1.410^{12}$ & $310^{-9}$ & 21 & - & - & - & - & - \\
IRAS 22272+5435 & $0.7^{\mathrm{c}}$ & $1.810^{16}$ & $6.910^{9}$ & 0.00029 & $1.510^{3}$ & - & 1 & - & $<0.01$ & 0.56 \\
AC Her & $0.2^{\mathrm{d}}$ & $4.310^{15}$ & $8.510^{10}$ & 0.0029 & $1.110^{5}$ & - & 1 & - & $<0.01$ & $1.110^{-4}$ \\
SAO 163075 & $0.5^{\mathrm{c}}$ & $7.510^{15}$ & $1.3510^{10}$ & 0.0099 & $5.310^{4}$ & - & 1 & - & $<0.001$ & 0.03 \\
AFGL 2688 & $0.8^{\mathrm{e}}$ & $1.410^{16}$ & $5.310^{9}$ & 0.0099 & $2.510^{5}$ & - & 1 & - & $<0.02$ & 0.6 \\
Red Rectangle & $2^{\mathrm{f}}$ & $1.110^{16}$ & $8.510^{8}$ & 0.077 & $1.310^{5}$ & $310^{2}$ & $<4$ & - & $<0.002$ & $2.510^{-5}$ \\
IRAS 21282+5050 & $1.4^{\mathrm{g}}$ & $6.310^{16}$ & $1.710^{9}$ & 0.75 & $2.010^{5}$ & $510^{4}$ & 1 & 0.008 & 0.1 & 2.7 \\
AFGL 618 & $0.4^{\mathrm{h}}$ & $1.010^{16}$ & $2.110^{10}$ & 0.75 & $4.410^{7}$ & $10^{6}$ & 1 & $410^{-4}$ & $0.01^{*}$ & 1.6 \\
NGC 6720 & $29^{\mathrm{i}}$ & $3.910^{17}$ & $4.010^{6}$ & 0.99 & $1.310^{3}$ & $510^{2}$ & 2.5 & 0.2 & $0.06^{* *}$ & 0.34 \\
NGC 7027 & $4^{\mathrm{j}}$ & $6.010^{16}$ & $2.110^{8}$ & 0.99 & $5.510^{5}$ & $10^{6}$ & 1 & 0.04 & 0.2 & 1.4 \\
\hline
\end{tabular}

* Questionable detection.

** Mass of the observed portion of the nebula.

References: (a) Skinner et al. 1998; (b) assumed values (few stellar radii); (c) Meixner et al. 1997; (d) Shenton et al. 1992; Alcolea \& Bujarrabal 1991; (e) Cox et al. 2000; Skinner et al. 1997; (f) Meixner et al. 1999; (g) Likkel et al. 1994; Meixner et al. 1993; (h) Kwok \& Bignell 1984; (i) Kastner et al. 1994; (j) Graham et al. 1993.

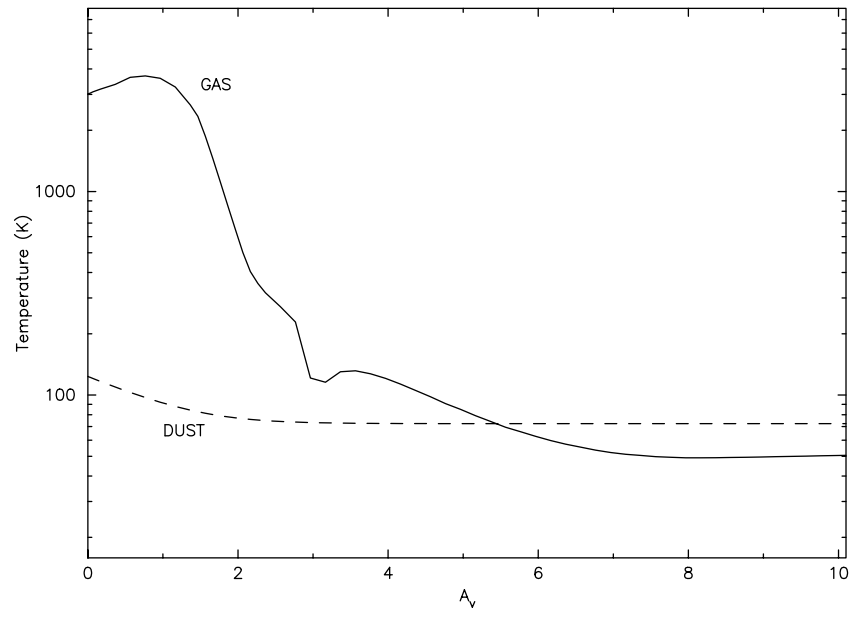

Fig. 7. The gas and dust temperature profiles in the PDR model, for $G=10^{5} G_{0}$ and $n=10^{5} \mathrm{~cm}^{-3}$

near the surface of the PDR, is the best way to determine this radius. For two sources, NGC 7027 and NGC 6720, we adopted the average radius of the $\mathrm{H}_{2}$ emission. If an $\mathrm{H}_{2}$ image can not be found, then we assume that the PDR is sandwiched between the inner $\mathrm{H}$ II region and the outer molecular envelope. The inner PDR radius can then be determined either from the inner edge of a CO line map (e.g. AFGL 2688) or the periphery of a radio continuum image (e.g. AFGL 618 or IRAS $21282+5050$ ). In the case of a PPN, where there is no ionized gas and the CO image does not resolve the inner radius, then the inner PDR radius is taken to be the inner edge of the dust shell (e.g. IRAS 22272+5435). For AGB stars we took a standard value of $\sim 510^{14} \mathrm{~cm}$ for the inner PDR radius, which corresponds to the typical extent of the dust condensation radius.

To compare our data with the theoretical models, we must convert our measured line fluxes to line intensities. In all of our sources, except NGC 6720, the ISO aperture covers the entire region of interest. Thus, for this conversion we divide the flux by $4 \pi \theta^{2}$ where $\theta$ is the angular radius of the photodissociation region (Table 7; see Paper II for derivation). We make an exception for NGC 6720 because our single pointing does not cover the entire PDR emission region (see Sect. 3.2). For NGC 6720, we choose to divide the flux by $\pi \theta^{2}$ since the entire nebula was not covered. In addition to our FIR line intensity data, the [C I] $609 \mu \mathrm{m}$ line intensities are plotted for those sources which have reported detections (see Knapp et al. 2000 for a compilation). To convert the [CI] $609 \mu \mathrm{m}$ line intensities reported in $\mathrm{K} \mathrm{km} \mathrm{s}^{-1}$ to erg $\mathrm{cm}^{-2} \mathrm{~s}^{-1} \mathrm{sr}^{-1}$, we first multiplied the reported values by $1.2210^{-7}$ (cf. Meixner et al. 1992, Appendix A). For AFGL 618, AFGL 2688 and the Red Rectangle, we then multiplied this intensity by $\pi \times\left(15^{\prime \prime} / 2\right)^{2} / 4 \pi \theta^{2}$ to account for the beam dilution in the same way we did for the ISO lines. For NGC 6720, the [C I] line emission is spatially resolved, so it does not need this correction.

The observed FIR line intensities are plotted as filled circles. Open circles indicate the upper limits. When FIR lines were detected with both the grating and FP spectrometers, the grating detections were plotted because their flux calibrations were more reliable. For the upper limits there were two cases: non-detections and detections in both the $\mathrm{ON}$ and OFF positions. For the 


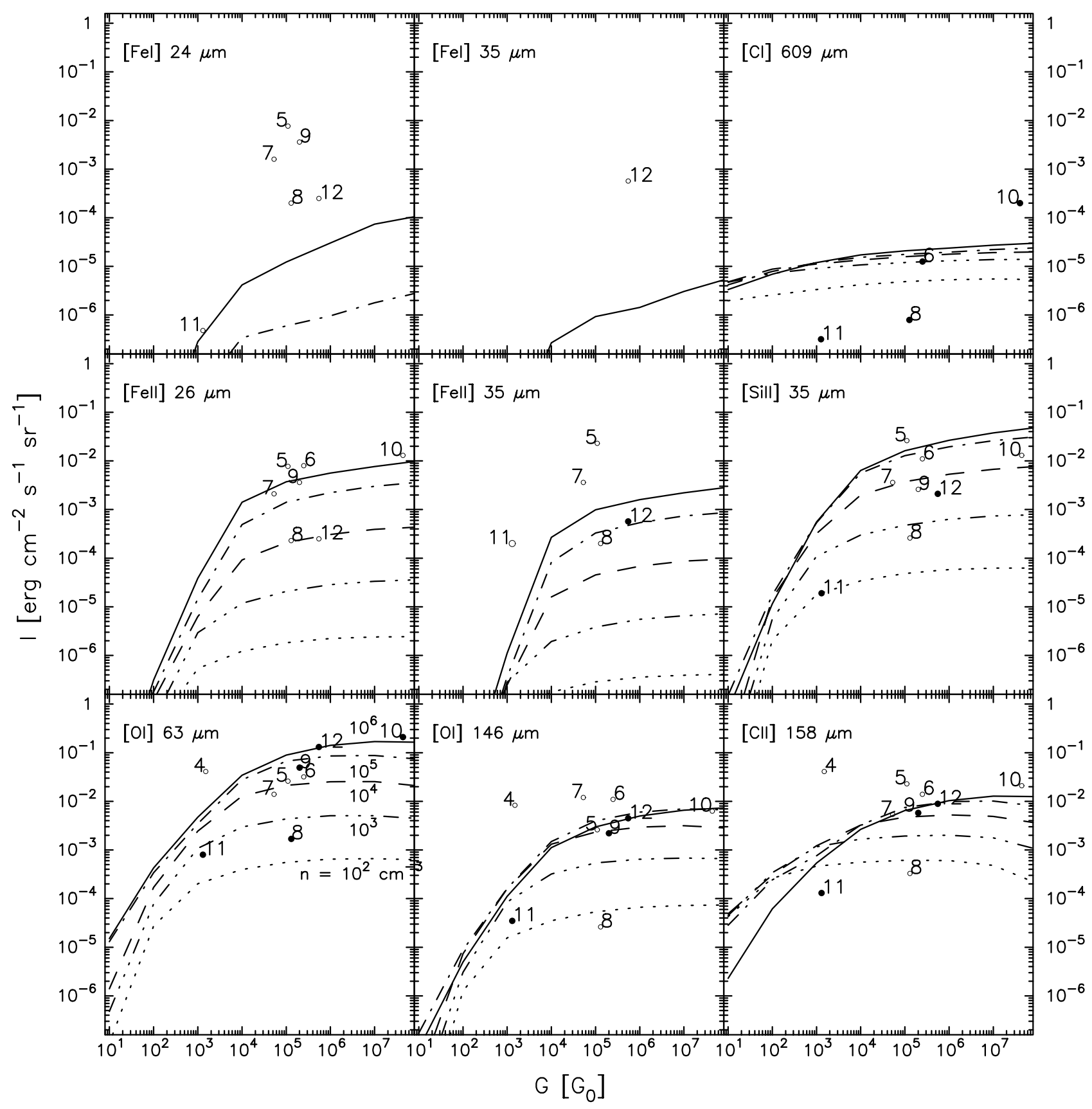

Fig. 8. Comparison of the observed fine-structure atomic lines with the PDR model. The curves represent the predicted intensities for models with densities from $10^{2}$ to $10^{6} \mathrm{~cm}^{-3}$. The filled circles indicate detections and the open circles indicate upper limits. The numeric symbols represent: 4-IRAS 22272+5435, 5-AC Her, 6-AFGL 2688, 7-SAO 163075, 8-Red Rectangle, 9-IRAS 21282+5050, 10-AFGL 618, 11-NGC 6720 and 12-NGC 7027

non-detections, $3 \sigma$ upper limits were plotted. For detections in both the $\mathrm{ON}$ and $\mathrm{OFF}$ scans, we plotted $2 \times(\mathrm{ON}-$ $\mathrm{OFF})$. For sources near the galactic plane with no OFF scans, we choose not to plot the intensities because we have no way to evaluate their reliability.

The general agreement between observations and theory is good, affirming the PDR origin of these FIR lines. The [O I] 63 and $146 \mu \mathrm{m}$ and [C II] $158 \mu \mathrm{m}$ lines were predicted to be the most intense lines and indeed, the observations clearly support that. The predicted intensities of neutral species like [FeI] are low because they will be largely ionized near the surface layers of the PDR (Fig. 6). Thus, our non-detections of [Fe I] in all of our sources are consistent with the model calculations. The ionized [Fe II] line was only detected in NGC 7027, the source with the highest $T_{\text {eff }}$ in the C-rich sample. In addition, [Si II] was only detected in NGC 6720 and NGC 7027, our 2 most evolved sources. Despite the predicted high abundance of $\mathrm{C}$ (Fig. 6), the intensity of [C I] $609 \mu \mathrm{m}$ is predicted to be lower compared to the other lines because of its smaller 
critical density and lower excitation energy. Nevertheless, ground based observations are sensitive enough to measure [C I].

For the case of NGC 7027, the source with the most lines detections, we can also directly compare the predicted line ratios to the observed line ratios. The predicted versus observed line ratios of $[\mathrm{O}$ I] 145/[O I]63 and [O I $] 63 /[\mathrm{CII}]$ are fairly consistent. [Fe II] $/[\mathrm{C} \mathrm{II}]$ only differs by a factor of 2 . However, the observed [Si II $] /[\mathrm{C}$ II $]$ is an order of magnitude lower than the model calculations. It is curious to find that the observed [Si II] intensity appears so depressed. A similar $[\mathrm{Si} \mathrm{II}] /[\mathrm{C} \mathrm{II}]$ ratio of $\sim 1 / 4$ is characteristic for reflection nebulae such as NGC 2023 (Steiman-Cameron et al. 1997). In contrast, the Orion Bar has a ratio of 1.5 (Tielens \& Hollenbach 1985b). This difference between the reflection nebulae and $\mathrm{H}$ II regions likely indicates a lower temperature and density for the former type of sources which can suppress the [Si II] line. However, that is unlikely the case for NGC 7027, where the PDR is known to be dense $\left(>10^{5} \mathrm{~cm}^{-3}\right)$ and warm (>1000 K; Hollenbach and Tielens 1999; Justtanont et al. 2000). Rather, this must reflect the elemental abundances in the gas. Perhaps most of the elemental silicon is condensed out in $\mathrm{SiC}$ grains in this source. Even though there is no sign of the $11.3 \mu \mathrm{m} \mathrm{SiC}$ dust feature in its midIR spectrum, it could be masked by the PAH emission (Beintema et al. 1996).

\subsection{Densities}

Density estimates of the low-excitation atomic gas in the PDR have been read straight off the PDR plots (Fig. 8) and are listed in Table 7 . The density curves tend to merge together at higher densities $\left(n>10^{5} \mathrm{~cm}^{-3}\right)$. When the closely spaced curves present confusion, the lowest density curve was taken. The density determinations are relatively sensitive to the observed intensities, but are rather insensitive to $G$, because the data points usually fall where the curves plateau. The densities are probably good to a factor of a few. For example, in the case of our best studied object, NGC 7027, the density determined from the [O I] and $[\mathrm{C}$ II $]$ lines are consistent at $\sim 10^{6} \mathrm{~cm}^{-3}$, our reported value. While the $[\mathrm{Fe} I \mathrm{I}]$ and $[\mathrm{SiI}]$ lines give densities that are $\sim 10 \times$ and $\sim 300 \times$ lower, respectively, these lower values may reflect depletion, as in the case of Si, instead of a lower density. Other models of NGC 7027 reveal similarly high densities. Latter et al. (2000) found that a density of $\sim 510^{4} \mathrm{~cm}^{-3}$ was consistent with the observed $\mathrm{H}_{2}$ emission characteristics. Yan et al. (1999) used $n=210^{6} \mathrm{~cm}^{-3}$ in the PDR model to fit the observed molecular rotational data.

\subsection{The effects of $G$ vs. $T_{\text {eff }}$}

PDR theory predicts that there should be strong atomic emission for sources with high $G$, so it is puzzling to find that there appears to be no correlation between line detections and $G$. However, there exists a relation between detections and $T_{\text {eff }} . G$ depends on $T_{\text {eff }}$ through $F_{\mathrm{UV}}$, the fraction of photons with energies higher than $6 \mathrm{eV}$ emitted by the central star (Table 7, see Paper II for details). Correcting for $F_{\mathrm{UV}}$ accounts for the photon energies needed to photoeject electrons off small grains (1st ionization potential $\sim 7 \mathrm{eV}$ for a PAH with $\sim 20 \mathrm{C}$ atoms; BT94), but not the fraction of photons with energies $>11 \mathrm{eV}$, the energy to photodissociate $\mathrm{CO}$ and to photo-ionize $\mathrm{C}$. Therefore in cooler stars, there will be too few photons with sufficient energy to form the atomic gas. Because it is necessary to photodissociate $\mathrm{CO}$ in order to create $\mathrm{O}$ in C-rich objects, non-detections of the [O I] line may indicate that $\mathrm{CO}$ photodissociation is not yet substantial.

Another factor to consider is the $\mathrm{CO}$ photodissociation lifetime as a function of $T_{\text {eff }}$ and $G$ (see Spaans et al. 1994, Fig. 3). The timescales to photodissociate CO are particularly important because atomic $\mathrm{C}$ and $\mathrm{O}$ dominate the cooling. For stars cooler than $10000 \mathrm{~K}$ and with $G \sim 10^{5}$ the photodissociation lifetime of CO is $\sim 100-1000$ years. This calculation holds for unattenuated radiation fields, so the lifetimes are lower limits in those regions (Tielens 1992). For AFGL 2688, with $T_{\text {eff }} \sim 7000 \mathrm{~K}$, the expected CO lifetime is $\sim 200$ yr. However, based on the adopted inner shell radius $\left(1.410^{16} \mathrm{~cm}\right.$, Table 7$)$ and the nebular expansion velocity $\left(15 \mathrm{~km} \mathrm{~s}^{-1}\right.$ for the AGB wind excluding the high velocity outflow), the nebula left the AGB phase only about 300 years ago. Furthermore, it is clear that during most of this time the stellar temperature was lower than the present value of $7000 \mathrm{~K}$. Therefore, it is very probable that objects with stellar temperatures similar to AFGL 2688 did not have time, during their evolution off the AGB branch, to significantly photodissociate the molecular gas. This also could explain the low atomic line emission observed in such objects.

Now there is a slight puzzle for the C-rich evolved star sample because the [CI] $609 \mu \mathrm{m}$ line has been detected in two of our objects with $T_{\text {eff }}<10000 \mathrm{~K}, \mathrm{IRC}+10216$ (Keene et al. 1993) and AFGL 2688 (Young 1997). Why is the $\left[\mathrm{CI}_{\mathrm{I}}\right]$ line detected but not the $[\mathrm{O} \mathrm{I}]$ or $\left[\mathrm{CII}_{\mathrm{II}}\right]$ lines? In the case of $\mathrm{IRC}+10216$, the $[\mathrm{C}$ I] emission may be explained by the photodissociation of acetylene $\left(\mathrm{C}_{2} \mathrm{H}_{2}\right)$ and $\mathrm{CO}$ in the outer circumstellar envelope by the interstellar radiation field (Keene et al. 1993). Outside of the CO photodissociation radius, we could expect there to be a significant column of $\mathrm{C}^{+}$and $\mathrm{O}$ atoms. Thus, our lack of a [C II] $158 \mu \mathrm{m}$ or [O I] $63 \mu \mathrm{m}$ line detection suggests that the densities and temperature of the gas in the outer envelope of IRC +10216 are too low to excite these lines. If they are to be observed at all, these lines are more likely to be detected in absorption against the central continuum source (Glassgold, private communication).

In the case of AFGL 2688, it has been suggested that the $\left[\mathrm{C}_{\mathrm{I}}\right]$ line emission may be shock dissociatied (Knapp et al. 2000). However, the line width of the [C I] line is comparable to the slow moving $\mathrm{CO}$ component in this source, suggesting a more quiescent origin from a PDR. An alternative explanation is that the [C I] line in AFGL 2688 
arises from the photodissociation of $\mathrm{C}_{2}$ which in turn is a product of $\mathrm{C}_{2} \mathrm{H}_{2}$ photodissociation (cf. Sect. 5.4). The minimum energy to photodissociate $\mathrm{C}_{2}$ is $6.2 \mathrm{eV}$ (Huber \& Herzberg 1979) which is significantly lower than the corresponding threshold for CO. AFGL 2688's central star has a $T_{\text {eff }}$ of $7000 \mathrm{~K}$ and thus, approximately $1 \%$ of its bolometric luminosity $\left(2.5 \quad 10^{4} L_{\odot}\right)$ lies above this minimum energy making this explanation plausible (see Table 7). The reason we do not detect $[\mathrm{CII}]$ or $[\mathrm{OI}]$ is not because of insufficient excitation conditions, as in the case of IRC +10216 ; these inner regions are sufficiently warm and dense. It is also not a lack of sensitivity due to beam dilution because the angular size of AFGL 2688's PDR is not significantly smaller than that of IRAS $21282+5050$ for which we unambiguously detect [C II] (see Table 7). The most likely explanation is just the low abundance of the $\mathrm{C}^{+}$and O. Thus, AFGL 2688 contains a PDR, but one too cool to photodissociate $\mathrm{CO}$ and to ionize $\mathrm{C}$.

This is the first time that PDR models have been widely applied to C-rich circumstellar environments. The physics and the chemistry of these models are still not completely understood, so there is a degree of uncertainty in the model results. Our models assume plane parallel, steady-state and stationary PDRs. To sufficiently model circumstellar envelopes we need to incorporate spherical, time-dependent and non-stationary effects. The evolutionary timescales for PPNe are very short ( 1000 years), so the gas is quickly evolving during this transition phase. The luminosity remains fairly constant, but the effective temperature increases rapidly. The factor $F_{\mathrm{UV}}$ accounts for the fraction of $F_{\mathrm{UV}}$ photons emerging from the star, but it does not account for the energies nor timescales needed to photodissociate the molecules. Finally, a detailed model of a specific evolved star should take into account the specific elemental abundances for that source.

\section{Comparison with line emission theory for shocked regions}

Shocks can develop when the post-AGB fast wind (100-1000 $\mathrm{km} \mathrm{s}^{-1}$ ) encounters the slower AGB wind $\left(\sim 10 \mathrm{~km} \mathrm{~s}^{-1}\right)$ ejected during the previous epoch of mass loss. The wind driven shock waves compress, heat, accelerate and dissociate the ambient molecular gas. J-type shocks occur for shock velocities $>40-50 \mathrm{~km} \mathrm{~s}^{-1}$ in molecular gas. In J-shocks, the temperature and the density of the gas jump from their pre-shock to their post-shock values. The post-shock gas is heated to temperatures of a few times $10^{4} \mathrm{~K}$, which dissociates the molecular material. The emission from J-shocks is primarily in the visible and the UV, but some is in atomic fine structure lines (HM89). C-shocks are non-dissociative shocks that heat the gas to temperatures of a few $10^{3} \mathrm{~K}$; the post-shocked gas cools mainly through molecular emission. C-shocks are not expected to produce ionized species like [Fe II], [Si II] and $[\mathrm{C}$ II]. The detection of these species would suggests that a PDR or a J-shock is present (van den Ancker 1999).
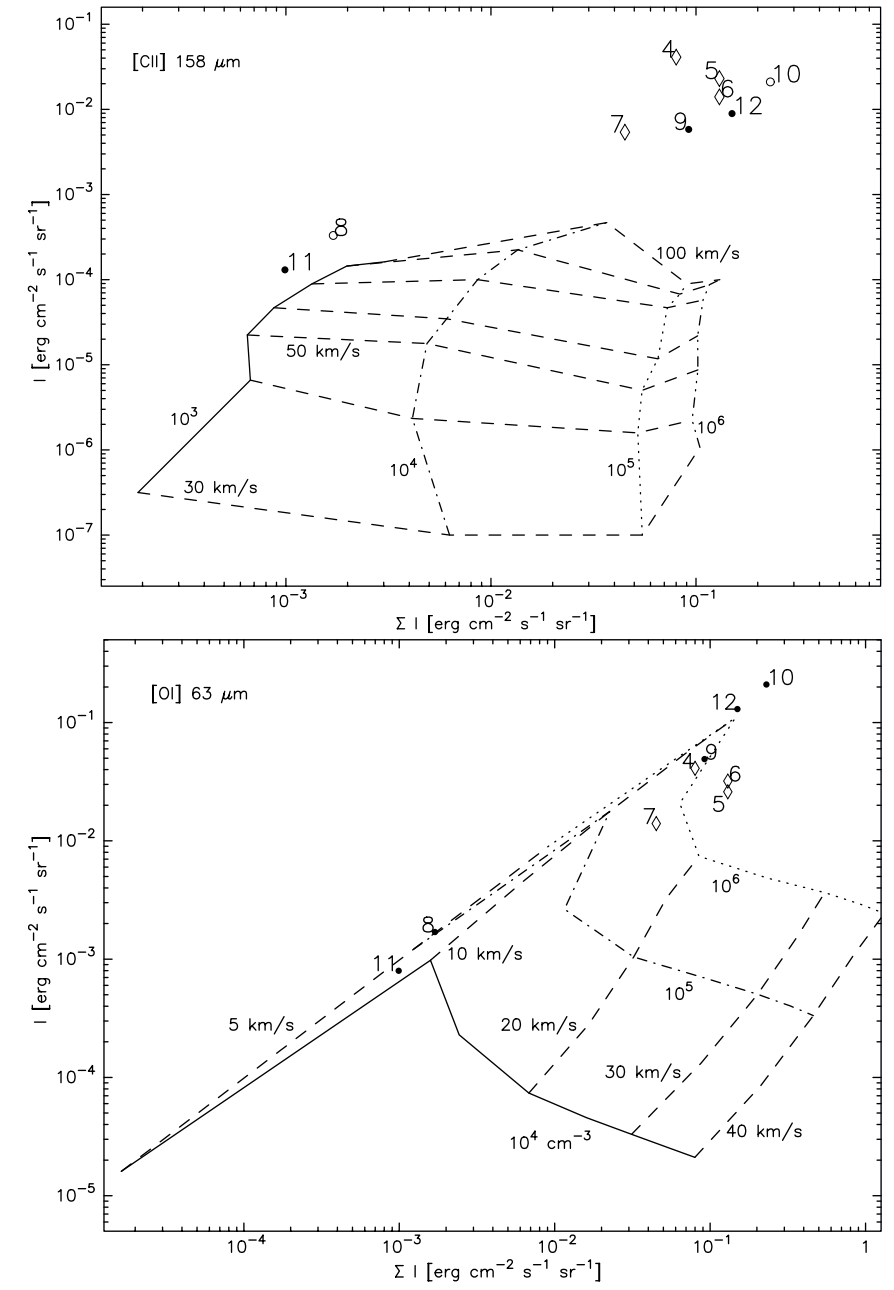

Fig. 9. Top: comparison of the observed [C II] $158 \mu \mathrm{m}$ line with J-shock models. The curves show predicted intensities for models with shock velocities of 40 to $100 \mathrm{~km} \mathrm{~s}^{-1}$ and preshock gas densities of $10^{3}$ to $10^{6} \mathrm{~cm}^{-3}$. Bottom: comparison of the observed [OI] $63 \mu \mathrm{m}$ line with C-shock models. The curves show predicted intensities for models with shock velocities of 5 to $40 \mathrm{~km} \mathrm{~s}^{-1}$ and pre-shock gas densities of $10^{4}$ to $10^{6} \mathrm{~cm}^{-3}$. $\Sigma \mathrm{I}$ is the summation of all the detected FIR line intensities. Filled circles signify detections, open circles represent upper limits in just the $y$-axis and diamonds indicate upper limits in both axes. The numeric symbols represent: 4-IRAS 22272+5435, 5-AC Her, 6-AFGL 2688, 7-SAO 163075, 8-Red Rectangle, 9-IRAS 21282+5050, 10AFGL 618, 11-NGC 6720 and 12-NGC 7027

We compare our observed line intensities to the predictions of both the J- and C-type shock models which are adapted from van den Ancker (1999) and HM89. These shock models are more appropriate for interstellar molecular clouds with O-rich chemistries, but we are applying their model predictions to our C-rich circumstellar case because C-rich shock models are not available. The shock model extends over the shock velocity and pre-shock gas density range of $v=40-100 \mathrm{~km} \mathrm{~s}^{-1}$ and $n=10^{3}-10^{6} \mathrm{~cm}^{-3}$ for J-shocks, and $v=$ $5-40 \mathrm{~km} \mathrm{~s}^{-1}$ and $n=10^{4}-10^{6} \mathrm{~cm}^{-3}$ for C-shocks. 
The J-shock models cover the same atomic cooling lines as given in the PDR models in addition to $[\mathrm{SI}$, while the C-shock models cover only the neutral [OI] and [S I] lines. For the J-shock model, only the [C II] line (Fig. 9) is shown because for the remaining FIR transitions, the model grid lines for the densities and velocities are too compressed together. For the C-shock model, we show only the [O I] $63 \mu \mathrm{m}$ line results (Fig. 9).

J-shocks do not appear to be present in the Red Rectangle, IRAS 21282+5050, AFGL 618, NGC 6720 or NGC 7027. The [C II] emission is predicted to be orders of magnitude lower in shocks than in PDRs. The observed [C II] emission generally lies about 2 orders of magnitude higher than the J-shock model predictions (Fig. 9). Although the $\mathrm{C}$ abundance is on average $\sim 5$ times higher in the C-rich sources, it is unlikely that abundances alone can account for the huge differences. J-shock models predict that the intensities for neutral species like Fe and $\mathrm{S}$ are orders of magnitude higher than in PDR models and hence be detectable. These neutral species were not detected in any of our sources.

C-shocks, on the other hand, may be present in AFGL 618. The [OI] $63 \mu \mathrm{m}$ line has a narrow component associated with a PDR and a broad component (see Sect. 3.2) that may be associated with post-shocked, accelerated gas. The [C II] detection is uncertain because no OFF position was taken (see Sect. 2.2). However, even if the detection is real, the $[\mathrm{C} \mathrm{II}]$ could well be explained by the PDR component. The broad component of the [OI] $63 \mu \mathrm{m}$ emission could be explained by a low velocity, high density C-shock (Fig. 9).

The vibrational transitions of $\mathrm{H}_{2}$ have also been used to identify shocks or PDRs in some of our sources. The $\mathrm{H}_{2}$ observations of AFGL 618 show signs that the $\mathrm{H}_{2}$ emission is dominated by shocks, but excitation from PDRs is also present (Hora et al. 1999). The $\mathrm{H}_{2}$ spectrum of NGC 7027 shows strong UV excitation, indicating a PDR (Latter et al. 2000; Hora et al. 1999; Latter et al. 1995). PDR models of the high J CO rotational lines by Yan et al. (1999) suggest the existence of shocked molecular gas in NGC 7027; however, Justtanont et al. (2000) can account for all the CO emission with a PDR model. $\mathrm{H}_{2}$ emission found predominantly in the outer lobes of IRAS $21282+5050$ also suggest the presence of shocks (Hora et al. 1999; Latter et al. 1995). Our atomic line analysis shows ample evidence for PDRs in all the sources, but only AFGL 618 shows signs of shock excitation.

\section{Atomic masses}

An important result of this work is the estimation of the PDR masses, especially for the PPNe since this is the first time that low-excitation atomic masses have been calculated for these transition objects. In this section, we use a restricted definition of $\mathrm{PDR}$ to mean that the $\mathrm{PDR}$ extends out to only the $\mathrm{C}^{+} / \mathrm{C} / \mathrm{CO}$ interface. The $[\mathrm{C}$ II] line emission serves as a good tracer for the low-excitation atomic regions. In these warm regions $\left(T_{\mathrm{k}}>100-200 \mathrm{~K}\right)$, the excitation temperature of $[\mathrm{C} \mathrm{II}]$ is much greater than its small excitation energy $(92 \mathrm{~K})$. [C II] is also expected to be optically thin. These conditions allow the observed line strength to be independent of the density and temperature, and dependent only on the emitting mass. The mass estimates of the PDR gas are calculated using the following equation which is derived in Paper II,

$$
M\left(M_{\odot}\right)=7.010^{6} F_{\mathrm{CII}}\left(\mathrm{erg} \mathrm{cm}{ }^{-2} \mathrm{~s}^{-1}\right) D(\mathrm{kpc})^{2} F_{\mathrm{c}} / X_{\mathrm{C}},
$$

where $F_{\mathrm{CII}}$ is the observed [C II] flux, $D$ is the distance, $F_{\mathrm{c}}$ is the correction factor when the excitation temperature is not very high (see Table 7 ) and $X_{\mathrm{C}}$ is the carbon abundance. Source specific carbon abundances were used for IRAS 22272+5435, AC Her, Red Rectangle, NGC 6720 and NGC 7027. Otherwise, when abundances were not available, an adopted average abundance of $1.510^{-3}$ was used (see Sect. 5.1).

The atomic mass estimates are listed in Table 7. Upper limits are given for the sources where [C II] was not detected. The PDR masses range from $0.01-0.2 M_{\odot}$. The mass estimate for AFGL 618 is uncertain because the [C II] line detection is questionable (see Sect. 2.2). For NGC 6720 , the ISO LWS beam covered only $\sim 1 / 3$ of the total nebular extent (see Sect. 3.2), thus the reported atomic mass corresponds to just the observed coverage.

Our atomic mass estimates are consistent with the values determined from previous studies. Our derived masses for IRAS 21282+5050, NGC 6720 and NGC 7027 are similar to those derived by Liu et al. (2001). Dinerstein (1991, $1995 \mathrm{a}, 1997)$ found that the PDR masses for $4 \mathrm{PNe}$, including NGC 7027, were about $0.1 M_{\odot}$, comparable to the mass of ionized gas in the nebulae. Ellis \& Werner (1985) also detected [C II] in NGC 7027 and derived an atomic mass $\sim 0.2 M_{\odot}$. Bachiller et al. (1994) detected $[\mathrm{CI}]$ in NGC 6720 and found that the mass of the neutral shell is $\gtrsim 0.1 M_{\odot}$.

For comparison, ionized and molecular mass estimates are listed in Table 7 . The ionized masses were taken from Huggins et al. (1996) and rescaled to our assumed distances. The molecular masses were derived from $\mathrm{CO}$ observations. The molecular masses for AC Her, Red Rectangle (Jura \& Kahane 1999), SAO 163075 (Bujarrabal et al. in preparation), NGC 6720 (Bachiller et al. 1989) and NGC 7027 (Jaminet et al. 1991) were taken from the literature and corrected to our adopted distances. When molecular masses were not available, then the mass loss rates, expansion velocities and radii of the $\mathrm{CO}$ shell for IRAS 22272+5435 (Loup et al. 1993), AFGL 2688 (Yamamura et al. 1996), IRAS 21282+5050 and AFGL 618 (Meixner et al. 1998) were used to estimate the mass loss time scale and to derive the molecular masses.

Comparisons of the low-excitation atomic masses to the molecular and ionized masses are more revealing for two reasons: 1) mass ratios will negate the distance uncertainties in the mass determinations and 2) the mass of ejected material depends on the mass of the progenitor star, hence comparing total masses can be misleading. The atomic to molecular mass ratio is 
about 0.04 for IRAS 21282+5050, 0.1 for NGC 7027 and 0.5 for NGC 6720 (after correcting by a factor of 3 to roughly account for the entire nebula). For the young $\mathrm{PNe}$ IRAS 21282+5050 and NGC 7027, the molecular component harbors most of the nebular mass, while the atomic component becomes more significant for the older PN NGC 6720. These results are consistent with the growing importance of the PDR as the PN evolves. The ionized to atomic mass ratio is about 0.08 for IRAS $21282+5050$, 0.2 for NGC 7027 and 1 for NGC 6720 (after correcting to account for the entire nebula). This ratio reveals that even for our most evolved source, the low-excitation atomic component is comparable to the mass of the ionized gas.

\section{Conclusions}

Atomic fine structure lines were observed in 12 C-rich objects spanning the AGB through the PN stage of evolution. Our analysis argues in favor of photodissociation as the major driving agent of chemical evolution. Combining our results with the 12 O-rich objects (Paper II), we find that the FIR line emission was detected only for the sources where the $T_{\text {eff }}>10000 \mathrm{~K}$. With higher $T_{\text {eff }}$ the number of detectable lines increase as well as the intensity of the emission, which imply that these atomic lines originate from PDRs.

New C-rich PDR models were presented for nine atomic cooling lines: [O I] 63 and $145 \mu \mathrm{m}$, [C II] $158 \mu \mathrm{m}$, [Si II] $35 \mu \mathrm{m},[\mathrm{Fe} \mathrm{I}] 24$ and $35 \mu \mathrm{m}$, [Fe II] 26 and $35 \mu \mathrm{m}$ and [C I] $609 \mu \mathrm{m}$. The predicted line intensities sufficiently match the observations, which allowed the density of the emitting layers to be estimated. The shock models do not compare well with the observed line intensities.

In AFGL 618, the broad velocity component seen in the [O I] $63 \mu \mathrm{m}$ line imply post-shocked, accelerated gas. However, the kinematics of the atomic gas in the rest of our sample are comparable to the kinematics of the ambient molecular gas seen in ${ }^{12} \mathrm{CO}$. These conditions are expected from cooling lines associated with circumstellar PDRs.

PDR mass estimates ranging from $\sim 0.01-0.2$ $M_{\odot}$ were derived from the [C II] $158 \mu \mathrm{m}$ line emission. When compared to the associated molecular masses, the atomic gas occupies only a small fraction of the total mass for young PNe, but grows significantly as the PN evolves.

Acknowledgements. This work has been financially supported by NASA JPL 961504 and NASA NAG 5-3350. Meixner has been additionally supported by NSF AST-97-33697. CastroCarrizo and Bujarrabal has been supported by Spanish DGES, under project PB96-0104. Kelly has also been supported by USAF Contract No. F19628-93-K-0011. Latter and Tielens acknowledge additional support from NASA grant 399-20-61 from the Long Term Space Astrophysics Program. We thank A. Glassgold, M. Haas, J. Lacy, Q. Nguyen-Rieu, and C. Skinner for discussions on the observations. The ISO Spectral Analysis Package (ISAP) is a joint development by the LWS and SWS Instrument Teams and Data Centers. Contributing institutes
Table 8. [Ne II] Line fluxes

\begin{tabular}{lc}
\hline Source & $\begin{array}{c}{[\mathrm{Ne} \mathrm{II}] \text { Flux }} \\
\left(10^{-13} \mathrm{erg} \mathrm{cm}^{-2} \mathrm{~s}^{-1}\right)\end{array}$ \\
\hline BD+303639 & $3410 \pm 30$ \\
IC 418 & $3300 \pm 200$ \\
NGC 2346 & $<3$ \\
U Monoceros & $<2$ \\
OH 231.8+4.2 & $<2$ \\
IRAS 07134+1005 & $<0.8$ \\
Frosty Leo & $<0.7$ \\
\hline
\end{tabular}

are CESR, IAS, IPAC, MPE, RAL and SRON. OSIA is a joint development of the SWS consortium. Contributing institutes are SRON, MPE, KUL and the ESA Astrophysics Division.

\section{Appendix A: Ground-based observations}

[Ne II] $12.8 \mu \mathrm{m}$ line fluxes were measured on 27-28 December 1995 using the mid-infrared echelle spectrometer Irshell (Lacy et al. 1989; Achtermann 1994a) at the NASA Infrared Telescope Facility. The spectrometer used an $11 \times 64 \mathrm{Si}$ :As array. The 2 -pixel slitwidth provided a resolution of roughly 9000. The platescale was $1 \mathrm{arcsec} /$ pixel. Data were collected in telescope scanning mode, which involved drifting the $11^{\prime \prime}$ long slit of the spectrometer back and forth across the nebulae while clocking out the array at $60 \mathrm{~Hz}$. A drift rate of $1 \mathrm{arcsec} / \mathrm{s}$ was used, and data were coadded by 60 . The result is an $11 \times 64 \times Z$ data cube, where $Z$ is the scan length in arcseconds. Bright reference stars were observed using the same procedures.

Data were reduced using the custom software program Snoopy (Achtermann 1994b). Data were dark subtracted and bad pixels were rejected. Frames with cloud contamination were identified either by their DC levels or by their atmospheric line strengths and were rejected. A dometemperature card, chopped against the sky, was used for flatfielding, atmospheric correction, and flux calibration (Lacy et al. 1989).

An infrared sky frame was obtained by linearly interpolating between the sky measurements before and after scanning across the nebulae. We compared our standard star fluxes to published photometry and found that we systematically underestimated the stellar fluxes by a factor of 1.45. This difference arises because astronomical sources provide a different solid angle to the array than does our flux calibration card, which is located just outside the window to the dewar. We therefore multiplied all of our card-calibrated nebular data by 1.45 to correct this flux underestimate.

Errors are estimated from the residuals of line fits and from the variation of line fits of individual scans. Uncorrected flatfield ripples in the continuum dominate over shot noise errors in several of the brighter nebulae. Line fluxes for detected sources were determined by summing the spectra from all slit and scan positions that showed source flux. Single or multiple Gaussians were fit 
to the resulting spectra using the package gaussfit (Jefferys 1990). Systematic errors are also possible and add an additional $5-10 \%$ of uncertainty above the listed errors.

In continuum sources without detectable line flux, we binned over all spatial positions in which the continuum was detected at a 2-sigma level or greater. The spectra were then binned by a number of pixels equal to the fullwidth half-maximum of the point spread function. The rms of these binned spectra was taken to be the 1-sigma upper limit to the line flux. The rough accuracy of these upper limits was confirmed by adding Gaussians to the data and determining visually what strength of Gaussian needed to be added to the data to provide a 3-sigma detection. The estimates from these two techniques tended to be consistent within $20 \%$.

In addition to our [Ne II] measurements, we also looked for [Cl I] $882.4 \mathrm{~cm}^{-1}$ and $\mathrm{H}_{2} J=4-2,814.4 \mathrm{~cm}^{-1}$ lines in AFGL 618. Neither was detected, with $3 \sigma$ upper limits of $1.010^{-12}$ and $710^{-12} \mathrm{erg} \mathrm{s}^{-1} \mathrm{~cm}^{-2}$ respectively.

The $[\mathrm{Ne} I \mathrm{I}]$ observations of objects with no ISO data are reported in Table 8 for completeness.

\section{References}

Achtermann, J. M. 1994a, PASP, 106, 173

Achtermann, J. M. 1994b, Ph.D. Thesis, University of Texas Aitken, D. K., \& Roche, P. F. 1982, MNRAS, 200, 217

Alcolea, J., \& Bujarrabal, V. 1991, A\&A, 245, 499

Bagnulo, S., Doyle, J. G., \& Griffin, I. P. 1995, A\&A, 301, 501

Barlow, M. J. 1999, Proceedings of the IAU Symposium 191 on AGB Stars, ed. T. Le Betre, A. Lebre, \& C. Waelkens (ASP: San Francisco), 353

Bachiller, R., Huggins, P. J., Cox, P., \& Forveille, T. 1994, A\&A, 281, L93

Bachiller, R., Bujarrabal, V., Martin-Pintado, J., \& GomezGonzalez, J. 1989, A\&A, 218, 252

Bakes, E. L. O., \& Tielens, A. G. G. M. 1994, ApJ, 427, 822 (BT94)

Beintema, D. A., van den Ancker, M. E., Molster, F. J., et al. 1996, A\&A, 315L, 369

Bujarrabal, V., Gómez-González, J., Bachiller, R., \& MartínPintado, J. 1988, A\&A, 204, 242

Bujarrabal, V., Alcolea, J., \& Planesas, P. 1992, A\&A, 257, 701

Bujarrabal, V., Castro-Carrizo, A., Alcolea, J., \& Sanchez Contreras, C. 2000, A\&A, in preparation

Cahn, J. H. 1984, ApJ, 279, 304

Calvet, N., \& Cohen, M. 1978, MNRAS, 182, 687

Castro-Carrizo, A., Bujarrabal, V., Fong, D., et al. 2001, A\&A, 367,674

Cernicharo, J., Guelin, M., Penalver, J., Martin-Pintado, J., \& Mauersberger, R. 1989, A\&A, 222L, 1

Cernicharo, J., Liu, X.-W., Gonzalez-Alfonso, E., et al. 1997, ApJ, 483L, 65

Clegg, P. E., Ade, P. A. R., Armand, C., et al. 1996, A\&A, 315, L38

Cohen, M., \& Hitchon, K. 1996, AJ, 111, 962

Cohen, M., Allamandola, L., Tielens, A. G. G. M., et al. 1986, ApJ, 302, 737

Cohen, M., Tielens, A. G. G. M., \& Bregman, J. D. 1989, ApJ, $344 \mathrm{~L}, 13$
Cox, P., Gonzalez-Alfonso, E., Barlow, M. J., et al. 1996, A\&A, 315,265

Cox, P., Lucas, R., Huggins, P. J., et al. 2000, A\&A, in press

Crowther, P. A., De Marco, O., \& Barlow, M. J. 1998, MNRAS, 296, 367

de Graauw, T., Haser, L. N., Beintema, D. A., et al. 1996, A\&A, 315, L49

Dinerstein, H. L. 1991, PASP, 103, 861

Dinerstein, H. L., Sneden, C., Uglum, J. 1995a, ApJ, 447, 262

Dinerstein, H. L., Haas, M. R., Erickson, E. F., \& Werner, M. W. 1995b, in Airborne Astronomy Symposium on the Galactic Ecosystem, ed. M. R. Haas, J. A. Davidson, \& E. F. Erickson, ASP Conf. Ser., 73, 365

Dinerstein, H. L., Haas, M. R., Erickson, E. F., \& Werner, M. W. 1997, Planetary Nebulae, Proceedings of the IAU Symposium 180, ed. H. J. Habing, \& H. J. G. L. M. Lamers (Dordrecht: Kluwer Academic Publishers), 220

Draine, B. T. 1978, ApJS, 36, 595

Ellis, H. B., \& Werner, M. W. 1985, Mass Loss from Red Giants, ed. M. Morris, \& B. Zuckerman (Dordrecht: Reidel Publishing Co.), 309

Feuchtgruber, H., Lutz, D., Beintema, D. A., et al. 1997, ApJ, 487, 962

Feuchtgruber, H. 1998, Status of SWS Fabry-Perot wavelength calibration and instrumental profile, ESA ISO Data Centre

Gammie, C. F., Knapp, G. R., Young, K., Phillips, T. G., \& Falgarone, E. 1989, ApJ, 345, 87

Giridhar, S., Lambert, D. L., \& González, G. 1998, ApJ, 509, 366

Graham, J. R., Serabyn, E., Herbst, J. M., et al. 1993, AJ, 105, 250

Groenewegen, M. A. T. 1997, A\&A, 317, 503

Groenewegen, M. A. T., van der Veen, W. E. C. J., \& Matthews, H. E. 1998a, A\&A, 338, 491

Groenewegen, M. A. T., Whitelock, P. A., Smith, C. H., \& Kerschbaum, F. 1998b, MNRAS, 293, 18

Gustafsson, B., Eriksson, K., Kiselman, D., Olander, N., \& Olofsson, H. 1997, A\&A, 318, 535

Habing, H. J. 1968, Bull. Astr. Inst. Netherlands, 19, 421

Herpin, F., \& Cernicharo, J. 2000, ApJ, 530, 129

Hollenbach, D., \& McKee, C. F. 1989, ApJ, 342, 306 (HM89)

Hollenbach, D. J., \& Tielens, A. G. G. M. 1997, ARA\&A, 35, 179

Hollenbach, D. J., \& Tielens, A. G. G. M. 1999, Rev. Modern Phys., 71, 173

Hora, J. L., Latter, W. B., \& Deutsch, L. K. 1999, ApJS, 124, 195

Hrivnak, B. J., Kwok, S., \& Volk, K. M. 1989, ApJ, 346, 265

Hrivnak, B. J. 1995, ApJ, 438, 341

Huber, K. P, \& Herzberg, G. 1979, Molecular Spectra and Molecular Structure IV. Constants of Diatomic Molecules (Van Nostrand Reinhold Co., New York), 112

Huggins, P. J., Bachiller, R., Cox, P., \& Forveille, T. 1996, A\&A, 315, 284

Iben, I. 1983, ApJ, 275, 65

Iben, I. 1995, Phys. Rep., 250, 2

Jaminet, P. A., Danchi, W. C., Sutton, E. C., et al. 1991, ApJ, 380,461

Jefferys, W., McArthur, B., \& McCartney, J. 1990, BAAS, 22, 927

Johnson, H. R., Ensman, L. M., Alexander, D. R., et al. 1995, ApJ, 443, 281

Jourdain de Muizon, M., Cox, P., \& Lequeux, J. 1990, A\&AS, 83,337 
Jura, M., \& Kahane, C. 1999, ApJ, 521, 302

Justtanont, K., Barlow, M. J., Tielens, A. G. G. M., et al. 2000 $\mathrm{A} \& \mathrm{~A}$, in press

Kastner, J. H., Gatley, I., Merrill, K. M., Probst, R., \& Weintranb, D. 1994, ApJ, 421, 600

Kaufman, M. J., \& Neufeld, D. A. 1996, ApJ, 456, 611

Kaufman, M. J., Wolfire, M. G., Hollenbach, D. J., \& Luhman, M. L. 1999, ApJ, 527, 795

Keene, J., Young, K., Phillips, T. G., Buettgenbach, T. H., \& Carlstrom, J. E. 1993, ApJ, 415, 131

Kelly, D. M., \& Latter, W. B. 1995, AJ, 109, 1320

Kessler, M. F., Steinz, J. A., Anderegg, M. E., et al. 1996, A\&A, 315, L27

Kholopov, P. N. 1985, General Catalogue of Variable Stars, the fourth edition

Knapp, G. R., \& Morris, M. 1985, ApJ, 292, 640

Knapp, G. R., Bowers, P. F., Young, K., \& Phillips, T. G. 1995, ApJ, 455, 293

Knapp, G. R., Crosas, M., Young, K., \& Ivezic, Z. 2000, ApJ, 534,324

Kwitter, K. B., \& Henry, R. B. C. 1998, ApJ, 493, 247

Kwok, S., \& Bignell, R. C. 1984, ApJ, 276, 544

Kwok, S. 2000, Asymmetrical Planetary Nebulae II: From Origins to Microstructures, ASP Conf. Ser. 199, ed. J. H. Kastner, N. Soker, \& S. Rappaport, 9

Lacy, J. H., Achtermann, J. M., Bruce, D. E., et al. 1989, PASP, 101,1166

Latter, W. B., Kelly, D. M., Hora, J. L., \& Deutsch, L. K. 1995 ApJS, 100, 159

Latter, W. B., Dayal, A., Bieging, J. H., et al. 2000, ApJ, in press

Latter, W. B., \& Tielens, A. G. G. M., in preparation

Le Bertre, T. 1997, A\&A, 324, 1059

Leech, K., de Graauw, T., \& van den Ancker, M. 2000, ISO Handbook, vol. VI: SWS - The Short Wavelength Spectrometer, ESA ISO Data Centre

Likkel, L., Morris, M., Kastner, J. H., \& Forveille, J. 1994, A\&A, 282, 190

Liu, X.-W., Barlow, M. J., Nguyen-Q-Rieu, et al. 1996, A\&A, 315, L257

Liu, X.-W. 1997, Proc. First ISO Workshop on Analytical Spectroscopy, ed. A. M. Heras, K. Leech, N. R. Trams, \& M. Perry, ESA SP-419, 87

Liu, X.-W., Barlow, M. J., Cohen, M., et al. 2001, MNRAS, in press

Loup, C., Forveille, T., Omont, A., \& Paul, J. 1993, A\&AS, 99, 291

Mathis, J. S., Rumpl, W., \& Nordsieck, K. H. 1977, ApJ, 217, 425

Meixner, M., Haas, M. R., Tielens, A. G. G. M., Erickson, E. F., \& Werner, M. 1992, ApJ, 390, 499

Meixner, M., Skinner, C. J., Temi, P., et al. 1993, ApJ, 411, 266
Meixner, M., Skinner, C. J., Graham, J. R., et al. 1997, ApJ, 482,897

Meixner, M., Campbell, M. T., Welch, W. J., \& Likkel, L. 1998, ApJ, 509, 392

Meixner, M., Ueta, T., Dayal, A., et al. 1999, ApJS, 122, 221

Middlemass, D. 1990, MNRAS, 244, 294

Pequignot, D., Baluteau, J.-P., Morisset, C., \& Boisson, C. 1997, A\&A, 323, 217

Press, W. T., Teukolsky, S. A., Vetterling, W. T., \& Flannery, B. P. 1992, Numerical Recipes in Fortran 77, 2nd edition (Cambridge Univ. Press), 684

Ryde, N., Schoeier, F. L., \& Oloffson, H. 1999, A\&A, 345, 841

Shenton, M., Albinson, J. S., Barrett, P., et al. 1992, A\&A, 262,138

Skinner, C. J., Meixner, M., Barlow, M. J., et al. 1997, A\&A, 328,290

Skinner, C. J., Meixner, M., \& Bobrowsky, M. 1998, MNRAS, 300, L29

Spaans, M., Tielens, A. G. G. M., van Dishoeck, E. F., \& Bakes, E. L. O. 1994, ApJ, 437, 270

Steiman-Cameron, T., Haas, M. R., Tielens, A. G. G. M., \& Burton, M. G. 1997, ApJS, 478, 261

Sternberg, A., \& Dalgarno, A. 1995, ApJS, 99, 565

Swinyard, B. M., Burgdorf, M. J., Clegg, P. E., et al. 1998, SPIE, 3354, 888

Szczerba, R., Omont, A., Volk, K., Cox, P., \& Kwok, S. 1997, A\&A, 317, 859

Taylor, A. R., Gussie, G. T., \& Pottasch, S. 1990, ApJ, 351, 515

Tielens, A. G. G. M., \& Hollenbach, D. 1985a, ApJ, 291, 722 (TH85)

Tielens, A. G. G. M., \& Hollenbach, D. 1985b, ApJ, 291, 747

Tielens, A. G. G. M. 1992, Planetary Nebulae, Proceedings of the IAU Symposium 155, ed. R. Weinberger, \& A. Acker (Dordrecht: Kluwer Academic Publishers), 157

van den Ancker, M. 1999, Ph.D. Thesis, University of Amsterdam

Van Winckel, H., Waelkens, C., \& Waters, L. B. F. M. 1995, A\&A, 293, L25

Van Winckel, H., \& Reyniers, M. 2000, A\&A, 354, 135

Waelkens, C., Van Winckel, H., Trams, N. R., \& Waters, L. B. F. M. 1992, A\&A, 256, 15

Waters, L. B. F. M., Cami, J., de Jong, T., et al. 1998, Nature, 391,868

Yamamura, I., Onaka, T., Kamijo, F., Deguchi, S., \& Ukita, N. 1996, ApJ, 465, 926

Yan, M., Federman, S. R., Dalgarno, A., \& Bjorkman, J. E. 1999, ApJ, 515, 640

Young, K. 1997, ApJ, 488, L157

Young, K., Keene, J., Phillips, T. G., Betz, A. L., \& Borieko, R. T. 1999, ApJ, submitted

Zacs, L., Klochkova, V. G. \& Panchuk, V. E. 1995, MNRAS, 275,764 